\title{
SELECTED TOPICS ON REACTION-DIFFUSION-ADVECTION MODELS FROM SPATIAL ECOLOGY
}

\author{
KING-YEUNG LAM， SHUANG LIU， YUAN LOU
}

\begin{abstract}
We discuss the effects of movement and spatial heterogeneity on population dynamics via reaction-diffusion-advection models, focusing on the persistence, competition, and evolution of organisms in spatially heterogeneous environments. Topics include LoktaVolterra competition models, river models, evolution of biased movement, phytoplankton growth, and spatial spread of epidemic disease. Open problems and conjectures are presented. Parts of this survey are adopted from the materials in [89, 108, 109, and some very recent progress are also included.
\end{abstract}

\section{Introduction}

Recent years have witnessed unprecedented progress of experimental technologies in the life sciences. The explosion of empirical results have rapidly generated massive sets of loosely structured data. The analytical methods from mathematics and statistics are required to synthesize the large data sets and extract insightful information from them. This trend continues to accelerate the development in mathematical biology. The study of mathematical biology usually includes two aspects. On the one hand, by introducing and analyzing mathematical models, researchers can elucidate and predict the basic mechanisms of underlying biological processes. On the other hand, deeper understanding of these mechanisms may drive the discovery of new mathematical problems, new analytical techniques, and even launch new research directions. Most, if not all, areas of mathematics have found applications in mathematical biology.

As an important mathematical area, nonlinear partial differential equations have been one of the most active research fields in the 21st century. It has also found new opportunities in mathematical biology in recent years. In [47] Dr. Avner Friedman discussed some challenging problems arising from mathematical biology, and one of the major mathematical tools he used is nonlinear partial differential equations. The subject of partial differential equations concerns the changes of quantities in space and time. For biology, the importance of space is hardly a question. With mathematics, we can endeavor to quantify the effect of space in different biological scenarios. In this survey, we consider some issues in spatial ecology via reactiondiffusion models, focusing upon the effect of movement of species on population dynamics in spatially heterogeneous environments. An important ongoing trend in population dynamics is the integration with other directions of biosciences, such as evolution, epidemiology, cell biology, cancer research, etc. Our main goal is to showcase the role of reaction-diffusion models by integrating across different research directions in mathematical biology, including spatial ecology, evolution and disease transmissions. We will illustrate that the quantitative analysis

2010 Mathematics Subject Classification. 35K57, 92D25, 92D40, 92D30, 37N25.

Key words and phrases. Spatial ecology, reaction-diffusion-advection models, population dynamics.

K.-Y. Lam, Y. Lou: Department of Mathematics, Ohio State University, Columbus, OH 43210, USA.

S. Liu: Institute for Mathematical Sciences, Renmin University of China, Beijing 100872, China. 
of some important questions in biosciences does bring interesting and novel mathematical problems, which in turn calls for the development of new mathematical tools. We will also formulate a number of potential research questions along the way.

This paper is organized as follows. In Section 2 we discuss several single species models, for which the dynamics have important implications for the invasion of exotic species. Section 3 is devoted to the study of two-species competition models, with emphasis on the effects of dispersal and spatial heterogeneity on the outcome of competition for two similar species. In Section 4 we investigate the competition models with directed movement and show how different dispersal (either random or biased) can bring dramatic changes to population dynamics. Section 5 concerns the continuous trait models and evolution of dispersal. Finally, in Sections 6 and 7 we discuss some progress on dynamics of phytoplankton growth and disease transmissions, respectively.

\section{Single species models}

The study of mathematical models for single species has played a primary role in population dynamics. It is also crucial in analyzing the dynamics of multiple interacting species, e.g. on issues concerning the invasions of exotic species. In this section we will focus on two types of single species models and study the effect of varying the diffusion rate.

2.1. Logistic model. In this subsection, we consider the logistic model

$$
\begin{cases}\partial_{t} u=d \Delta u+u[m(x)-u] & (x, t) \in \Omega \times(0, \infty), \\ \frac{\partial u}{\partial \nu}=0 & (x, t) \in \partial \Omega \times(0, \infty), \\ u(x, 0)=u_{0}(x) & x \in \Omega,\end{cases}
$$

where $\Omega$ is a bounded domain in $\mathbb{R}^{N}$ with smooth boundary $\partial \Omega$ and $\nu(x)$ denotes the unit outward normal vector at $x \in \partial \Omega$. Here $u(x, t)$ represents the density of the single species at location $x$ and time $t$. Parameter $d>0$ is the diffusion rate and $\Delta=\sum_{i=1}^{N} \frac{\partial^{2}}{\partial x_{i}^{2}}$ is the usual Laplace operator. The function $m \in C^{2}(\bar{\Omega})$ accounts for the local carrying capacity or the intrinsic growth rate of the species, which is assumed to be strictly positive in $\bar{\Omega}$ for the sake of clarity. The Neumann boundary condition indicates that no individuals can move across $\partial \Omega$, i.e. the habitat is closed. We assume that the initial data $u_{0}$ is non-negative and not identically zero.

It is well known [12] that

$$
\lim _{t \rightarrow \infty} u(x, t)=u^{*}(x, d) \quad \text { uniformly for } x \in \bar{\Omega},
$$

where for each $d>0$, the function $u^{*}(\cdot, d)$ is the unique positive solution of

$$
\begin{cases}d \Delta u+u(m-u)=0 & x \in \Omega, \\ \frac{\partial u}{\partial \nu}=0 & x \in \partial \Omega .\end{cases}
$$

If the spatial environment is homogeneous, i.e. $m$ is a positive constant, then $u^{*}(x, d) \equiv m$ is the unique positive solution of (2), which is independent of the diffusion rate $d$. If the spatial environment is heterogeneous, i.e. $m=m(x)$ is a non-constant function, then $u^{*}(x, d)$ depends non-trivially on $d$. In this case, a natural question is how the total biomass of the species at equilibrium, i.e. $\int_{\Omega} u^{*}(x, d) \mathrm{d} x$, depends on the diffusion rate $d$.

The following property was observed in [105]: 
Lemma 2.1. If $m$ is non-constant, then for any diffusion rate $d>0$,

$$
\int_{\Omega} u^{*}>\int_{\Omega} m=\lim _{d \rightarrow 0} \int_{\Omega} u^{*}=\lim _{d \rightarrow \infty} \int_{\Omega} u^{*} .
$$

Lemma 2.1 means that (i) the heterogeneous environment can support a total biomass greater than the total carrying capacity of the environment, which is quite different from the homogeneous case; (ii) the total biomass, as a function of $d$, is non-monotone; it is maximized at some intermediate diffusion rate, and is minimized at $d=0$ and $d=\infty$, respectively. Examples were constructed in [98] to illustrate that this function can have two or more local maxima.

Lemma 2.1 implies that $\int_{\Omega} u^{*} / \int_{\Omega} m>1$ for all $d>0$, and the lower bound 1 is optimal. For the upper bound of $\int_{\Omega} u^{*} / \int_{\Omega} m$, Ni raised the following question:

Conjecture 1 ([130]). There exists some constant $C=C(N)$ depending only on $N$ such that

$$
\int_{\Omega} u^{*} / \int_{\Omega} m \leq C(N), \quad \text { and } \quad C(1)=3 .
$$

Conjecture 1 suggests that the supremum of the ratio of the biomass to the total carrying capacity depends only on the spatial dimension. For one-dimensional case, $C(1)=3$ was proved in [6] and it turns out to be optimal. However, it is recently shown by Inoue and Kuto [74] that Conjecture 1 fails for the higher-dimensional case even when $\Omega$ is a multi-dimensional ball. That is, the nonlinear operator which maps $m$ to $u^{*}$ is not bounded as a map from the positive cone of $L^{1}(\Omega)$ to itself.

A related question is the optimal control of total biomass [38]:

Optimization problem. Fix any $\delta \in(0,1)$, and define the control set

$$
U:=\left\{m \in L^{\infty}(\Omega): 0 \leq m \leq 1, \int_{\Omega} m=\delta|\Omega|\right\} .
$$

Determine those $m^{*} \in U$ which can maximize the total biomass $\int_{\Omega} u^{*}$ in $U$.

Nagahara and Yanagida [128] showed that, under a regularity assumption, the optimal $m^{*}$ is of "bang-bang" type, i.e. $m^{*}=\chi_{E}$ for some measurable set $E \subset \Omega$, where $\chi_{E}$ denotes the characteristic function of $E$. More recently, Mazari et al. [121] proved that the bang-bang property holds for all large enough diffusion rates. Biologically, the set $E$ can be viewed as the protected area, and the condition $\int_{\Omega} m=\delta|\Omega|$ means that the total resources are limited. The results in [121, 128] imply that under the limited resources, the way to maximize the total population size of species is to place all the resources evenly in some suitable subset $E$ of $\Omega$. However, the characterization of the set $E$ is a challenging problem. It seems that the problem has not been completely solved even in the one-dimensional case; see [79] for some progress on cylindrical domains. Recent work [127] for the discrete patch model suggests that the set $E$ is periodically fragmented; see also 123 for general fragmentation phenomenon. For the biased movement model with dispersal term $\nabla \cdot((1+m) \nabla u)$, the optimal distribution of resources for maximizing the survival ability of a species was considered in [122]. They showed that the problem has "regular" solutions only when the domain is a ball and the optimal distribution can be characterized in this case; see [11] for the one-dimensional case.

Compared to (2), a more general model is

$$
\begin{cases}d \Delta u+r(x) u\left(1-\frac{u}{K(x)}\right)=0 & x \in \Omega, \\ \frac{\partial u}{\partial \nu}=0 & x \in \partial \Omega\end{cases}
$$


where $r$ is the intrinsic growth rate and $K$ is the carrying capacity of environment. Is it possible to find general sufficient conditions for $r, K$ such that the maximal biomass size of species can be reached at some intermediate diffusion rate? This question for (3) was proposed and studied by DeAngelis et al. [35]. We refer to [59] for some relevant developments in this regard, which shows that the total biomass can be a monotone increasing function of the diffusion rate for some choices of $r$ and $K$.

In the study of a predator-prey model [113, the following problem arises: Is the maximum of the density in (2), i.e. $\max _{x \in \bar{\Omega}} u^{*}(x, d)$, monotone decreasing with respect to the diffusion rate $d$ ? Some recent progress is obtained in [97, where the monotonicity was proved for several classes of function $m$. However, it remains open to show the monotonicity for general function $m$. In contrast, the minimum of the density, i.e. $\min _{x \in \bar{\Omega}} u^{*}(x, d)$, is not necessarily monotone increasing in $d$; see 63]. We conjecture that the difference between the maximum and minimum values of the density, which measures the spatial variations of the density, is a monotone decreasing function of $d$. It is known that $\int_{\Omega}\left|\nabla u^{*}\right|^{2}$, which also measures the spatial variations of the density, is monotone decreasing with respect to $d$; see [98] for more details.

2.2. Single species models in rivers. How do populations persist in streams when they are constantly subject to downstream drift? This problem is intensified when the habitat quality at the downstream end is very poor. Once the species are washed downstream, the chances of survival will be greatly reduced. This problem, termed as the "drift paradox, has received considerable attention [65, 126]. Speirs and Gurney [144] pointed out that the action of dispersal can permit persistence in an advective environment, and they proposed the following reaction-diffusion-advection model:

$$
\begin{cases}\partial_{t} u=d \partial_{x x} u-\alpha \partial_{x} u+u(r-u) & x \in(0, L), t>0 \\ d \partial_{x} u(0, t)-\alpha u(0, t)=u(L, t)=0 & t>0, \\ u(x, 0)=u_{0}(x) & x \in(0, L),\end{cases}
$$

where positive constants $\alpha$ and $r$ are the advection rate and the growth rate of species, respectively. The interval $[0, L]$ represents an idealized river, assuming that the upstream end $x=0$ is a no-flux or closed boundary, while at the downstream end $x=L$ the zero Dirichlet boundary condition (also called lethal boundary condition) is imposed. Biologically interpreted, the downstream end describes the junction of rivers and seas, as freshwater fishes cannot survive in the seawater. The persistence of species is equivalent to the instability of the trivial equilibrium, which is in turn characterized by the negativity of the principal eigenvalue of the following problem:

$$
\left\{\begin{array}{l}
d \partial_{x x} \varphi-\alpha \partial_{x} \varphi+(r+\lambda) \varphi=0 \quad x \in(0, L), \\
d \partial_{x} \varphi(0)-\alpha \varphi(0)=\varphi(L)=0 .
\end{array}\right.
$$

By an explicit calculation, Speirs and Gurney [144] obtained the following necessary and sufficient condition for the persistence of species:

$$
d>\frac{\alpha^{2}}{4 r} \text { and } L>L^{*}(d):=\frac{\pi-\arctan \left(\sqrt{4 d r-\alpha^{2}} / \alpha\right)}{\sqrt{4 d r-\alpha^{2}} / 2 d} .
$$

Set $\underline{L}:=\inf _{d>0} L^{*}(d)$. As illustrated in Figure 1, condition (6) implies that

(i) if $L \leq \underline{L}$, then the species cannot persist for any diffusion rate $d$. In this case, the equilibrium $u=0$ is globally stable among all non-negative initial values; 


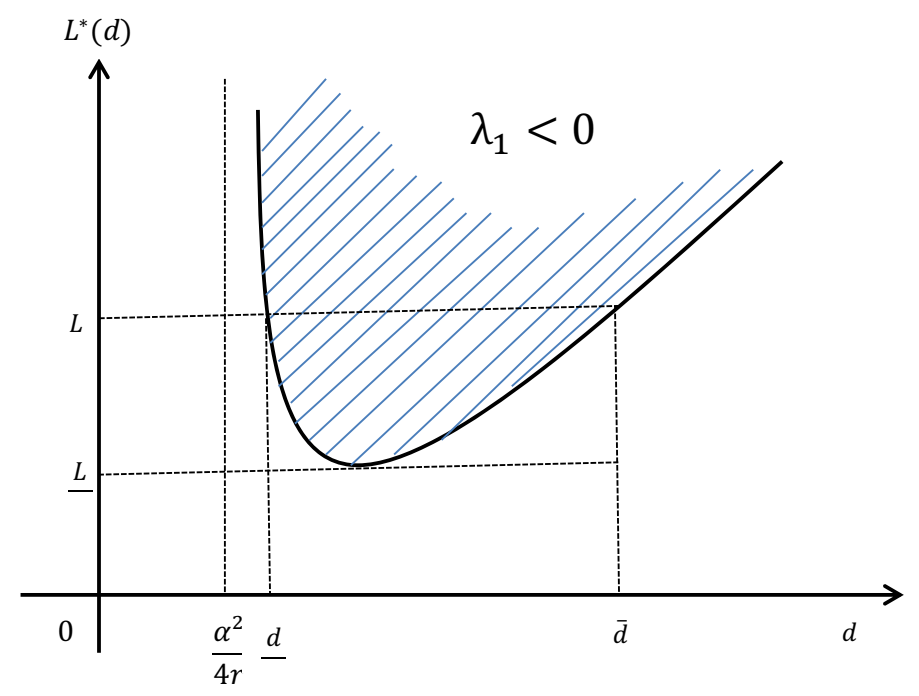

FiguRE 1 . The diagram of $L^{*}(d)$ illustrated by the black colored solid curve. The parameter region where the species persists is shaded in blue.

(ii) if $L>\underline{L}$, then there exist $0<\underline{d}<\bar{d}$ such that the species persists if and only if $d \in(\underline{d}, \overline{\bar{d}})$. Furthermore, if $d \notin(\underline{\underline{d}}, \bar{d})$, then $u=0$ is globally stable; if $d \in(\underline{d}, \bar{d})$ then (4) admits a unique positive steady state which is globally stable.

Biologically, the conditions for species survival are that the river has a minimal length and the diffusion rate of the species lies within a certain range: when the diffusion rate is small, the drift is so dominant that the species is washed downstream and cannot survive; when the diffusion rate is large, the species is exposed too much to the lethal boundary at the downstream end and will also go to extinction. Therefore, it was conjectured in [89, 110] that there is an intermediate diffusion rate $d^{*}$ which is the "best" strategy, i.e. once the diffusion rate of a species is $d^{*}$, no other species can replace it. This conjecture implies that if the majority of individuals in a population adopt the strategy $d^{*}$, then any invasion fails so long as the invading species is adopting a strategy different from $d^{*}$. In the evolutionary game theory, such $d^{*}$ is referred to as an evolutionary stable strategy [119]. See Subsection 3.2 and Conjecture 3 for further details.

In (4), the downstream end is assumed to be the lethal boundary. Recent work considering general boundary conditions [115, 148] showed that the species persists if and only if the velocity of flow drift is less than some threshold value. Under some other boundary conditions, this threshold value turns out to be a monotone decreasing function of the diffusion rate, while this is not the case for (4). This leads to a natural evolutionary question: Is the fast diffusion or slow diffusion more beneficial to the species in streams? We will discuss this question in Subsection 3.2. Some new persistence criteria has been found for general boundary conditions at the downstream in a most recent work [57], where the critical river length can be either monotone decreasing in the diffusion rate $d$ or it can first decrease and then increase as $d$ increases, depending on the loss rate at the downstream; see also [156] for related discussions. The diagram in Figure 1 can be regarded as an extreme case of the new persistence criteria found in [57]. 
Finally, we refer to [43, 78, 116, 117, 147, 151, 152] for related work on the persistence problem in rivers and river networks.

\section{Competing species models}

One basic question in spatial ecology is: How does the spatial heterogeneity of environment affect the invasion of exotic species and the competition outcome of native and exotic species? The term heterogeneity refers to the non-uniform distribution of various environmental conditions. For example, in the oceans, light intensity decreases with respect to the depth due to light absorption by phytoplankton and water [80], and thus is unevenly distributed. An exotic species can successfully invade often because it has some competitive advantage over the resident species. However, in the heterogeneous environment, with the help of diffusion alone, invasion is still possible even though the exotic species does not have any apparent competitive advantage. In this section, we consider some classical competition models to illustrate this phenomenon.

3.1. Lotka-Volterra competition models. We first consider the following Lotka-Volterra competition model in the homogeneous environment:

$$
\begin{cases}\partial_{t} u=d \Delta u+u\left(a_{1}-b_{1} u-c_{1} v\right) & (x, t) \in \Omega \times(0, \infty), \\ \partial_{t} u=D \Delta v+v\left(a_{2}-b_{2} u-c_{2} v\right) & (x, t) \in \Omega \times(0, \infty), \\ \frac{\partial u}{\partial \nu}=\frac{\partial v}{\partial \nu}=0 & (x, t) \in \partial \Omega \times(0, \infty), \\ u(x, 0)=u_{0}(x), v(x, 0)=v_{0}(x) & x \in \Omega,\end{cases}
$$

where $u(x, t)$ and $v(x, t)$ are the population densities of two competing species with diffusion rates $d$ and $D>0$, parameters $a_{1}, a_{2}$ are their intrinsic growth rates, $b_{1}, c_{2}$ are the intra-specific competition coefficients, and $b_{2}, c_{1}$ are the inter-specific competition coefficients. We assume for the moment that $a_{i}, b_{i}, c_{i}, i=1,2$, are positive constants, i.e. the spatial environment is homogeneous. If the coefficients satisfy the weak competition condition

$$
\frac{b_{1}}{b_{2}}>\frac{a_{1}}{a_{2}}>\frac{c_{1}}{c_{2}}
$$

then (4) admits a unique positive steady state $\left(u^{*}, v^{*}\right)$ given by

$$
u^{*}=\frac{a_{1} c_{2}-a_{2} c_{1}}{b_{1} c_{2}-b_{2} c_{1}}, \quad v^{*}=\frac{a_{2} b_{1}-a_{1} b_{2}}{b_{1} c_{2}-b_{2} c_{1}} .
$$

Theorem 3.1 ([10]). Suppose that the environment is homogeneous and (8) holds. Then the positive steady state $\left(u^{*}, v^{*}\right)$ is globally asymptotically stable among all non-negative and non-trivial initial conditions.

Theorem 3.1 shows that when the environment is homogeneous and the competition is weak, two competing species can always coexist regardless of the initial conditions. A natural question arises: Does Theorem 3.1 remain valid in spatially heterogeneous environment?

Obviously, when $a_{i}, b_{i}, c_{i}$ are non-constant functions in $\Omega,\left(u^{*}, v^{*}\right)$ given by $(9)$ is generally non-constant, and thus not necessarily a positive steady state of (7). However we may still ask: If condition (8) holds pointwisely on $\Omega$, does (7) admit a unique positive steady state which is globally asymptotically stable? Surprisingly, even if $(8)$ holds pointwisely in $\Omega$, it is possible that one of the two competing species eventually becomes extinct, regardless of initial conditions, provided that two competitors have proper diffusion rates. Mathematically, for the homogeneous case, (7) can be viewed as an ordinary differential equation, which has a 
globally stable positive steady state for each $x \in \Omega$. In contrast, for the heterogeneous case, the global attractor of the partial differential equation model (7) could simply be a semi-trivial steady state, so that the density of one competitor stays bounded from below while the other tends to zero, as $t \rightarrow \infty$. Thus, the spatial heterogeneity of environment also plays a crucial role here.

For further discussions on the competitive exclusion in spatially heterogeneous environment, we consider the following simplified competition model:

$$
\begin{cases}\partial_{t} u=d \Delta u+u[m(x)-u-b v] & (x, t) \in \Omega \times(0, \infty), \\ \partial_{t} v=D \Delta v+v[m(x)-c u-v] & (x, t) \in \Omega \times(0, \infty), \\ \frac{\partial u}{\partial \nu}=\frac{\partial v}{\partial \nu}=0 & (x, t) \in \partial \Omega \times(0, \infty), \\ u(x, 0)=u_{0}(x), v(x, 0)=v_{0}(x) & x \in \Omega,\end{cases}
$$

where $m(x)$ represents the common resource of two competitors and the weak competition condition (8) is reduced to $0<b, c<1$.

We assume that $m(x)$ is positive, smooth and non-constant to reflect the inhomogeneous distribution of resources across the habitat. Then for $d, D>0,(10)$ has two semi-trivial states, $\left(u^{*}, 0\right)$ and $\left(0, v^{*}\right)$, where $u^{*}$ is the unique positive solution of (2), while $v^{*}$ is the unique positive solution of (2) with $d$ replaced by $D$. For the spatially heterogeneous environment, the stability of semi-trivial states is a subtle question. For example, the stability of $\left(u^{*}, 0\right)$ is determined by the sign of the principal eigenvalue $\lambda_{1}$ of the problem

$$
\begin{cases}D \Delta \varphi+\left(m-c u^{*}\right) \varphi+\lambda \varphi=0 & x \in \Omega, \\ \frac{\partial \varphi}{\partial \nu}=0 & x \in \partial \Omega,\end{cases}
$$

where $u^{*}(x, d)$ depends implicitly on $d$. It is well-known [12 that if $\lambda_{1}>0$, then $\left(u^{*}, 0\right)$ is stable; if $\lambda_{1}<0$, then $\left(u^{*}, 0\right)$ is unstable. When $m$ is constant, under weak competition condition $0<b, c<1$, direct calculations give $\lambda_{1}<0$, i.e. $\left(u^{*}, 0\right)$ is always unstable for any $d, D>0$. In contrast, for non-constant $m$, the situation becomes complicated and interesting: First, the variational characterization of the principal eigenvalue [12 implies that $\lambda_{1}$ is monotone increasing with respect to $D$, and it tends to $\min _{\bar{\Omega}}\left(c u^{*}-m\right)$ as $D$ approaches 0 . Notice from (2) that $m-u^{*}$ must change sign in $\Omega$. If $c<1$ (in fact $c \leq 1$ is sufficient), then $\min _{\bar{\Omega}}\left(c u^{*}-m\right)<0$, which implies that $\lambda_{1}<0$ for small $D$. For large $\bar{D}$ we have

$$
\lim _{D \rightarrow \infty} \lambda_{1}=\frac{1}{|\Omega|}\left(c \int_{\Omega} u^{*}-\int_{\Omega} m\right),
$$

which highlights the role played by the total biomass $\int_{\Omega} u^{*}$ in the stability of $\left(u^{*}, 0\right)$. To be more precise, define

$$
c_{*}:=\inf _{d>0} \frac{\int_{\Omega} m}{\int_{\Omega} u^{*}} .
$$

From Subsection 2.1 we see that $c_{*} \in(0,1)$, and if $c<c_{*}$, then $\left(u^{*}, 0\right)$ is unstable for all $d, D>0$. It is interesting that when $c \in\left(c_{*}, 1\right)$, we can find some non-empty open set $\Sigma$ such that the semi-trivial solution $\left(u^{*}, 0\right)$ is stable if and only of $(d, D) \in \Sigma$, so that we can define

$$
\Sigma:=\left\{(d, D) \in(0, \infty) \times(0, \infty):\left(u^{*}, 0\right) \text { is stable }\right\} .
$$

Observe that for any $c \in\left(c_{*}, 1\right)$, the set $\Sigma$ is always a subset of $\{(d, D): 0<d<D\}$.

Recently, He and $\mathrm{Ni}$ [62] proved a surprising and insightful result: for a class of competition models, if the semi-trivial or positive solution is locally stable, then it must be globally stable. 
The following result, as a corollary of their general result, completely solves a conjecture proposed in [105, 107]:

Theorem 3.2. Suppose that $0<b \leq 1, c \in\left(c_{*}, 1\right)$ and $m$ is non-constant. If $(d, D) \in \Sigma$, then $\left(u^{*}, 0\right)$ is globally stable among all non-negative and non-trivial initial conditions; if $(d, D) \notin \bar{\Sigma}$ and $d<D$, then (10) admits a unique positive steady state which is globally stable.

Theorem 3.2 shows that for some diffusion rates, competition exclusion takes place even if the weak competition condition is satisfied everywhere in $\Omega$. The case $b=1$ and $c_{*}<c<1$ should be pointed out particularly. In this case, when the environment is homogeneous, i.e. $m$ is constant, the equilibrium solution $(0, m)$ is globally stable, so that the faster diffusing species excludes the slower one. In contrast, for the spatially heterogeneous environment, Theorem 3.2 implies that for proper diffusion coefficients, the final outcome of the competition is reversed: the slower diffusing species excludes the faster one!

Furthermore, when $b=1$ and $c$ approaches 1 from below, the following result holds:

Theorem 3.3. Assume that $b=c=1$. If $d<D$, then $\left(0, v^{*}\right)$ is unstable and $\left(u^{*}, 0\right)$ is globally stable. Conversely, if $d>D$, then $\left(0, v^{*}\right)$ is globally stable.

In fact, as $c$ tends to 1 from below, the set $\Sigma$ increases monotonically and converges to the set $\{(d, D): 0<d<D\}$ in the Hausdorff sense. Biologically, this implies that the slower diffusing species will replace the faster one in the spatially heterogeneous environment. This is the so-called evolution of slow dispersal. More precisely, if we consider the slower diffuser as a mutant among the faster diffusing population, then mutations for slower diffusion rate are selected, i.e. the diffusion rate of the "new resident" is smaller than the "previous resident". Through constant mutation and competition, the diffusion of new species thus becomes slower and slower. This phenomenon was first discovered by Hastings [58], and Theorem 3.3 was proved by Dockery et al. [39]. Moreover, it is shown in [71] that this result may fail for spatio-temporally varying environments; see also [104.

The above discussion illustrates that diffusion in a spatially heterogeneous environment has a dramatic effect on the dynamics of competition models, which is rather different from the case of a homogeneous environment. It should be noted that there are still many questions which remain open even for two competing species. We refer the interested readers to [23, 60, 61, 92, 129] for some related discussions. For general resource distributions, it is challenging to fully determine the stability of the semi-trivial state of (7); see [14, 70, 106] for examples of multiple reversals of the competition outcomes for two-species Lotka-Volterra competition models. A remaining problem is to find some conditions on the resource distribution, so that the local stability of two semi-trivial steady states of (7) can be accurately characterized. The answer may depend upon how to give some sufficient conditions ensuring that the total biomass of a single species as a function of diffusion rate has a unique local maximum (and thus also a global maximum). Another interesting question is what happens if all the coefficients $a_{i}, b_{i}, c_{i}$ in (7) are non-constant, and the weak competition condition (8) still holds for all $x \in \Omega$. See the recent progress in [131] via construction of special Lyapunov functions.

For other population models, such as the predator-prey models, we can enquire similar issues: How do diffusion and spatial heterogeneity affect the dynamics of predator-prey models? For the competition models, under the weak competition condition, if the diffusion rate of a species lies within some proper ranges, the remaining average resources can be negative, which is closely related to the phenomenon that the total biomass of a single species can be greater than the total amount of resources, as discussed in Subsection 2.1. Therefore, the fast diffusing invader cannot invade successfully in this case as their available resources can 
be negative. Similar consideration is applicable for the predator-prey models. However, the study of predator-prey models turns out to be more complicated [113]: Understanding the relationship between the total biomass of species and the total amount of resources is far from being sufficient, and other issues are also involved, such as the dependence of the maximum density of species on the diffusion rate 97.

The study of reaction-diffusion models not only uncover some interesting biological phenomena, but also bring new and interesting mathematical problems. In the next two subsections, we continue on the theme of competition, but focus on the effect of biased movement of species in the spatially heterogeneous environment.

3.2. Competition in rivers. In this subsection we consider the following two-species competition model in a stream:

$$
\begin{cases}\partial_{t} u=d \partial_{x x} u-\alpha_{1} \partial_{x} u+u(r-u-v) & (x, t) \in(0, L) \times(0, \infty), \\ \partial_{t} v=D \partial_{x x} v-\alpha_{2} \partial_{x} v+v(r-u-v) & (x, t) \in(0, L) \times(0, \infty), \\ d \partial_{x} u-\alpha_{1} u=D \partial_{x} v-\alpha_{2} v=0 & (x, t) \in\{0, L\} \times(0, \infty), \\ u(x, 0)=u_{0}(x), v(x, 0)=v_{0}(x) & x \in(0, L),\end{cases}
$$

where $\alpha_{1}$ and $\alpha_{2}$ represent the advection rates of species $u$ and $v$, respectively. When $\alpha_{1}=$ $\alpha_{2}=0$, it is shown in Subsection 3.1 that the species with the smaller diffusion rate will drive the other species to extinction, provided that $r$ is non-constant. What happens if $\alpha_{1}, \alpha_{2} \neq 0$ ?

For the case when $r$ is a positive constant (i.e. the environment is homogeneous) and $\alpha_{1}=\alpha_{2}=\alpha>0$, the following result was established in [115]:

Theorem 3.4. Suppose that $r>0$ is constant and $\alpha>0, D>d$. Then the semi-trivial state $\left(0, v^{*}\right)$ is globally stable, where $v^{*}$ is the unique positive solution of

$$
\begin{cases}D \partial_{x x} v-\alpha \partial_{x} v+v(r-v)=0 & x \in(0, L) \\ D \partial_{x} v-\alpha v=0 & x=0, L\end{cases}
$$

Theorem 3.4 shows that under the influence of passive drift, the species with faster diffusion will be favored, which is contrary to Theorem 3.3 for model $(10)$ in the non-advective environment. In the river, the "fate" of the two competitors is reversed: the slower diffusing species will be eventually wiped out! An intuitive reasoning is that the passive drift will push more individuals downstream to force a concentration of population at the downstream end, which causes mismatch of the population density with the resource distribution. Faster diffusion can counterbalance such mismatch by sending more individuals upstream and therefore, it is competitively advantageous for species to adopt faster diffusion.

When $r$ is constant, the case $\alpha_{1} \neq \alpha_{2}$ and $D=d$ was considered in [112], where it was proved that the smaller advection is always beneficial. A similar explanation is that under the drift, the species will move towards the downstream end, leading to the overcrowding and the overmatching of resources there, which in turn results in the extinction of the species with the stronger advection that can send more individuals to the downstream. The general case $D \neq d$ and $\alpha_{1} \neq \alpha_{2}$ was addressed in [158], which showed that the fast diffusion and weak advection will be favored, consistent with the findings in [112, 115].

When $r$ is non-constant, i.e. the spatial environment is heterogeneous, the dynamics of (13) turns out to be more complicated. The simplest situation might be when $r$ is decreasing, for which we have the following conjecture:

Conjecture 2. Suppose that $r$ is positive and decreasing in $(0, L)$. Then there exists some $\alpha^{*}>0$, independent of $d$ and $D$, such that 
(i) for $\alpha \in\left(0, \alpha^{*}\right)$, there exists $D^{*}=D^{*}(\alpha)>0$ such that if $D=D^{*}$ and $d \neq D^{*}$, then the semi-trivial steady state $\left(0, v^{*}\right)$ of $(13)$ is globally stable;

(ii) for $\alpha \geq \alpha^{*}$, if $D>d>0$, then $\left(0, v^{*}\right)$ of (13) is globally stable.

We observe that if $r$ is non-constant and $\alpha=0$, the slower diffusion rate is always favored. This suggests that $D^{*}(\alpha) \rightarrow 0$ as $\alpha \rightarrow 0$. Furthermore, we expect $D^{*}(\alpha) \rightarrow+\infty$ as $\alpha \rightarrow \alpha^{*}-$. We refer to [114, 157] for some recent developments for the case of decreasing $r$. The general case of $r(x)$ is studied in [90], where sufficient conditions for the existence of evolutionarily stable strategies, or that of branching points, are found. We refer the readers to [36] for the definition of a branching point, and to Subsection 4.2 for further discussions.

For the Speirs and Gurney model (4), it was conjectured that there is an intermediate diffusion rate which is optimal for the evolution of dispersal. This seems to be in contrast to Theorem 3.4. where the faster diffusion is favored. Such discrepancy is due to the different boundary conditions at the downstream end $x=L$; see [110, 115, 148] for further results on the effect of boundary conditions. Next, we impose the zero Dirichlet boundary conditions at the downstream end and consider the following competition model:

$$
\begin{cases}\partial_{t} u=d \partial_{x x} u-\alpha \partial_{x} u+u(r-u-v) & (x, t) \in(0, L) \times(0, \infty), \\ \partial_{t} v=D \partial_{x x} v-\alpha \partial_{x} v+v(r-u-v) & (x, t) \in(0, L) \times(0, \infty), \\ d \partial_{x} u-\alpha u=D \partial_{x} v-\alpha v=0 & (x, t) \in\{0\} \times(0, \infty), \\ u=v=0 & (x, t) \in\{L\} \times(0, \infty), \\ u(x, 0)=u_{0}(x), v(x, 0)=v_{0}(x) & x \in(0, L) .\end{cases}
$$

As shown in Subsection 2.2, neither large nor small diffusion rate is beneficial to each species in this case. A natural conjecture is:

Conjecture 3. There exists some $D^{*}(\alpha)>0$ such that if $D=D^{*}$ and $d \neq D^{*}$, the semitrivial steady state $\left(0, v^{*}\right)$ of $(15)$, whenever it exists, is globally stable.

For the case of weak advection ( $\alpha$ small), we conjecture that the approaches developed in [87, 88] may be applicable to yield the existence of $D^{*}$ for small $\alpha$, satisfying $D^{*}(\alpha) \rightarrow 0$ as $\alpha \rightarrow 0$. However, the proof remains quite challenging for general $\alpha$. Recently it was shown in [155] that such diffusion rate $D^{*}$ exists and is unique for two-patch models: The semi-trivial steady state $\left(0, v^{*}\right)$ is locally stable when $D=D^{*}$ and $d \neq D^{*}$. However, the global stability of $\left(0, v^{*}\right)$ remains unclear. For multi-patch models, it remains open to determine the existence of $D^{*}$.

\section{Competition models with directed movement}

Belgacem and Cosner [7] argued that in the spatially heterogeneous environment, the species may have a tendency to move upward along the resource gradient, in addition to random diffusion. In this connection, they added an advection term to (1) and considered the reactiondiffusion-advection model

$$
\begin{cases}\partial_{t} u=\nabla \cdot[d \nabla u-\alpha u \nabla m]+u(m-u) & (x, t) \in \Omega \times(0, \infty), \\ \frac{\partial u}{\partial \nu}-\alpha u \frac{\partial m}{\partial \nu}=0 & (x, t) \in \partial \Omega \times(0, \infty), \\ u(x, 0)=u_{0}(x) & x \in \Omega .\end{cases}
$$

Here $\alpha \geq 0$ is a parameter which measures the tendency of the population to move upward along the gradient of the resource $m(x)$. Belgacem and Cosner [7] and subsequent work [30] indicate that for a single species, the advection along the resource gradient can be beneficial 
to the persistence of the single species in convex habitats, but not necessary for non-convex $\Omega$. In particular, when $m$ is positive somewhere, the species persists whenever the advection rate $\alpha$ is large. The biological explanation is that the species can utilize the large advection to concentrate at places with locally most abundant resources.

It is natural to enquire whether the advection along the resource gradient can confer competition advantage. To this end, Cantrell et al. [15] proposed the following model:

$$
\begin{cases}\partial_{t} u=\nabla \cdot[d \nabla u-\alpha u \nabla m]+u(m-u-v) & (x, t) \in \Omega \times(0, \infty), \\ \partial_{t} v=D \Delta v+v(m-u-v) & (x, t) \in \Omega \times(0, \infty), \\ d \frac{\partial u}{\partial \nu}-\alpha u \frac{\partial m}{\partial \nu}=\frac{\partial v}{\partial \nu}=0 & (x, t) \in \partial \Omega \times(0, \infty), \\ u(x, 0)=u_{0}(x), v(x, 0)=v_{0}(x) & x \in \Omega .\end{cases}
$$

When $m$ is non-constant and $\int_{\Omega} m \geq 0,(17)$ has two semi-trivial states, denoted by $\left(u^{*}, 0\right)$ and $\left(0, v^{*}\right)$, for every $d, D>0$ and $\alpha \geq 0$ (see [30]), where $u^{*}$ is the unique positive steady state of (16). It is shown in [16] that if $d=D$ and $\Omega$ is convex, then the semi-trivial state $\left(u^{*}, 0\right)$ is globally stable for small $\alpha>0$. This implies that for two species which differ only in their dispersal mechanisms, the weak advection can confer some competition advantages, provided that the underlying habitat is convex. However, for some non-convex $\Omega$, it is also proved in [16] that the opposite case may happen for proper $m(x)$ : the weak advection can actually put the species at a disadvantage.

Does the species have a competitive advantage if the advection is strong? The following result of [5] might seem surprising at the first look. It was first proved in [16] under the additional assumption that the set of critical points is of measure zero and has at least one strict local maximum point.

Theorem 4.1. Suppose that $m \in C^{2}(\bar{\Omega})$ is positive and non-constant. Then for $\frac{\alpha}{d} \geq \frac{1}{\min _{\bar{\Omega}} m}$, both semi-trivial steady states $\left(u^{*}, 0\right)$ and $\left(0, v^{*}\right)$ are unstable, and (17) has at least one stable coexistence state.

Theorem 4.1 implies that the two competing species are able to coexist when the advection is strong, which is in stark contrast with the case of weak advection. What is preventing the species $u$ to exclude species $v$ by strong advection? A series of work suggests that as $\alpha$ increases, species $u$ resides only at the set $\mathfrak{M}$ of local maximum points of $m(x)$, and retreats from $\Omega \backslash \mathfrak{M}$. If $m$ is positive, then that leaves ample room for species $v$ to survive in $\Omega \backslash \mathfrak{M}$, i.e. places with less resources [16, 26, 83, 84, 91]. This is proved in Theorem 4.1. When $m(x)$ changes sign, however, the overall leftover resources in $\Omega \backslash \mathfrak{M}$ might be poor and $u$ may competitively exclude $v$ by strong advection.

Theorem $4.2(93])$. Suppose that $m \in C^{2}(\bar{\Omega})$ and it is sign-changing.

(a) If $\int_{\Omega \backslash \mathfrak{M}} m>0$, then for any $d, D>0$, u and $v$ coexist for large $\alpha$.

(b) If $\int_{\Omega \backslash \mathfrak{M}} m<0$, then for some $d, D>0, u$ competitively excludes $v$ for large $\alpha$.

Theorem 4.1 also reveals that the two competitors modeled by (17) can achieve coexistence by different dispersal strategies including random diffusion and directed advection towards resources, which suggests a new mechanism for coexistence of two or more competing species. In [111] we apply the similar ideas to discuss the possibility of coexistence for three-species competition systems with the random diffusion and directed advection. The analysis of threespecies models turns out to be much more difficult. An obvious challenge is that systems of three competing species, unlike two-species competition model, are not order-preserving [142]. 


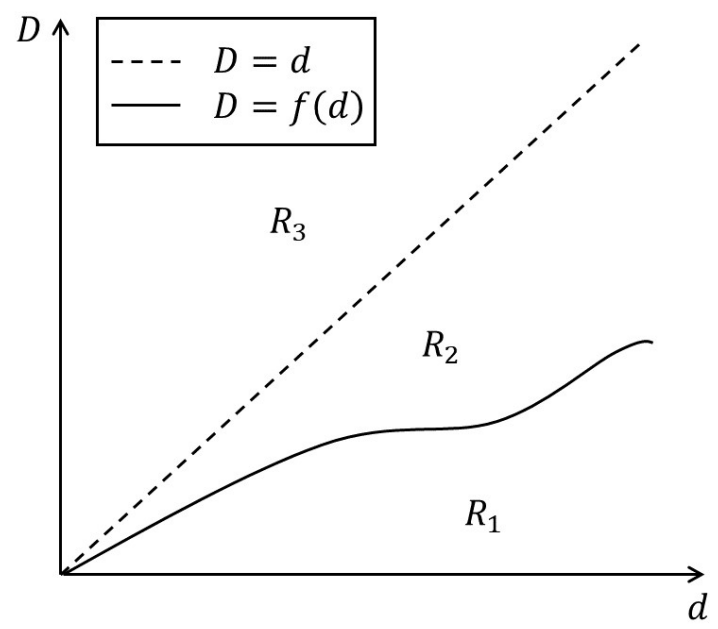

Figure 2. Decomposition of the $d-D$ parameter space into three regions: $R_{1}$ and $R_{2}$ are separated by the curve $D=f(d)$. The regions $R_{2}$ and $R_{3}$ are separated by the line $D=d$. The bifurcation diagrams for each of these regions, with $\alpha$ as the bifurcation parameter, are given in Figure 3 . (First published in Mem. Amer. Math. Soc. 245 (Spring 2017), published by the American Mathematical Society. @ 2017 American Mathematical Society.)

As mentioned earlier, if the advection rate $\alpha$ is large, then the species $u$ will concentrate near some local maximum points of $m$. Further studies [84, 91] showed that the concentration positions are precisely those local maximum points of $m$ where the function $m-v^{*}$ is also strictly positive, by establishing the uniform $L^{\infty}$ estimate and a new Liouville-type result. We note that $m$ is the common resource for both species $u$ and $v$, and $m-v^{*}$ is the resource available for species $u$ when the species $v$ reaches its equilibrium. It is observed in [91, Rmk. 1.4] that for some positive local maximum points of $m$, function $m-v^{*}$ can be negative, i.e. some apparently favorable places are actually dangerous ecological traps for the species $u$. This suggests that both distribution of resources and competitors should be taken into account when studying the directed movement of species along the resource gradient.

4.1. Global bifurcation diagrams. The above results show that when the advection is weak, the two species cannot coexist, and the advection confers some competitive advantage for species $u$; when the advection is strong, the two species coexist for any diffusion rates. What happens for general advection rates? For instance, assuming $d>D$, when we increase the advection from weak to strong, how does that affect the dynamics of (17)? If $\alpha=0$, the result of Dockery et al. 39 says that the semi-trivial state $\left(0, v^{*}\right)$ is globally stable; if $\alpha$ is sufficiently large, Theorem 4.1 shows that both $\left(u^{*}, 0\right)$ and $\left(0, v^{*}\right)$ are unstable and the coexistence happens. A possible conjecture is there exists some critical advection rate $\alpha^{*}>0$ such that for $\alpha<\alpha^{*},\left(0, v^{*}\right)$ is globally stable, while for $\alpha>\alpha^{*}$, (17) has a unique positive steady state which is globally stable. However this conjecture fails in general and a more complicated bifurcation diagram of positive steady states occurs. To be more precise, Averill et al. [5] showed that under some conditions on $m(x)$, there exists a function $f(x):[0, \infty) \rightarrow$ $[0, \infty)$ satisfying $f(0)=0$ and $0<f(x)<x$ such that the $d$-D parameter space is divided into three regions $R_{1}, R_{2}, R_{3}$, as shown in Figure 2. Using $\alpha$ as a bifurcation parameter, we see that the following three situations occur: 
(1) for $D<f(d)$, there exists some critical value $\alpha_{1}>0$ such that as $\alpha<\alpha_{1}$, the semitrivial state $\left(0, v^{*}\right)$ is stable but $\left(u^{*}, 0\right)$ is unstable, while as $\alpha>\alpha_{1}$, both $\left(u^{*}, 0\right)$ and $\left(0, v^{*}\right)$ are unstable so that (17) has at least one positive steady state. By the bifurcation theory, there is a branch of positive steady states, denoted by $\Gamma_{1}$, which bifurcates from $\left(0, v^{*}\right)$ at $\alpha=\alpha_{1}$ and can be extended to infinity in $\alpha$. This is illustrated in Figure 3(a). It is unclear whether $\left(0, v^{*}\right)$ is globally stable when $\alpha<\alpha_{1}$, and whether the positive steady state is unique and globally stable when $\alpha>\alpha_{1}$;

(2) for $f(d)<D<d$, there exist three critical values $0<\alpha_{1}<\alpha_{2}<\alpha_{3}$ such that (i) as $\alpha<\alpha_{1},\left(0, v^{*}\right)$ is stable but $\left(u^{*}, 0\right)$ is unstable; (ii) as $\alpha_{2}<\alpha<\alpha_{3}$, ( $\left.u^{*}, 0\right)$ is stable but $\left(0, v^{*}\right)$ is unstable; (iii) as $\alpha \in\left(\alpha_{1}, \alpha_{2}\right) \cup\left(\alpha_{3}, \infty\right)$, both $\left(u^{*}, 0\right)$ and $\left(0, v^{*}\right)$ are unstable so that (17) has at least one positive steady state. Similarly, by the bifurcation theory, there is still a branch of positive steady states bifurcating from $\left(0, v^{*}\right)$ at $\alpha=\alpha_{1}$, which is denoted as $\Gamma_{2}$. The difference is that the branch $\Gamma_{2}$ cannot be extended to $\alpha=\infty$, but is connected to $\left(u^{*}, 0\right)$ at $\alpha=\alpha_{2}$. Interestingly, when $\alpha \in\left(\alpha_{2}, \alpha_{3}\right)$, the semi-trivial state $\left(u^{*}, 0\right)$ is stable, which indicates that the advection can indeed be beneficial to the species in this case! When $\alpha>\alpha_{3}$, the branch of a positive steady state, denoted as $\Gamma_{3}$, bifurcates from $\left(u^{*}, 0\right)$ at $\alpha=\alpha_{3}$, and can be extended to $\alpha=\infty$. We conjecture that as $\alpha<\alpha_{1},\left(0, v^{*}\right)$ is globally stable; as $\alpha_{2}<\alpha<\alpha_{3},\left(u^{*}, 0\right)$ is globally stable; as $\alpha \in\left(\alpha_{1}, \alpha_{2}\right) \cup\left(\alpha_{3}, \infty\right)$, 17) admits a unique positive steady state which is globally stable. This is illustrated in Figure $3(b)$.

(3) for $D>d$, there exists some critical value $\alpha_{1}>0$ such that as $\alpha<\alpha_{1},\left(u^{*}, 0\right)$ is stable, while as $\alpha>\alpha_{1}$, both $\left(u^{*}, 0\right)$ and $\left(0, v^{*}\right)$ are unstable so that (17) has at least one positive steady state. Similarly, a branch of positive steady states bifurcates from $\left(u^{*}, 0\right)$ at $\alpha=\alpha_{1}$, denoted as $\Gamma_{4}$, which can be extended to $\alpha=\infty$. It is conjectured that $\left(u^{*}, 0\right)$ is globally stable when $\alpha<\alpha_{1}$, and the positive steady state is unique when $\alpha>\alpha_{1}$. This is illustrated in Figure $3(\mathrm{c})$.

How, then, is the branch $\Gamma_{1}$ transformed to $\Gamma_{2}$ and $\Gamma_{3}$, and finally to $\Gamma_{4}$, as the diffusion rate $D$ varies, with $d$ being fixed? The result in [5] shows that in the critical situation $D=f(d)$, the branch $\Gamma_{1}$ is connected to the semi-trivial state $\left(u^{*}, 0\right)$ at $\alpha=\alpha^{*}$ for some $\alpha^{*}>0$. Once $D>f(d), \alpha^{*}$ is split into two different points $\alpha_{2}$ and $\alpha_{3}$, so that $\Gamma_{1}$ is split into two disjoint branches $\Gamma_{2}$ and $\Gamma_{3}$. As $D$ approaches $d$ from below, the bounded branch $\Gamma_{2}$ will approach $\alpha=0$ and vanishes; meanwhile $\Gamma_{3}$ approaches $\Gamma_{4}$.

By these discussions, we explained that for the models with multiple parameters, it is possible to apply the bifurcation theory to describe the structure of positive steady states. Of course, it remains challenging to completely characterize the global dynamics of (17), and many interesting mathematical questions, e.g. uniqueness and global stability, are left open [5]. One particular conjecture is that, for $\alpha$ sufficiently large, the positive steady state of the competition system (17) is unique and thus globally asymptotically stable. One way of proving it is to determine the precise limiting profile and show that each positive steady state is linearly stable, whenever it exists. One can then conclude the uniqueness and global asymptotic stability by the theory of monotone dynamical system [64]. This was carried out for the case when all critical points of $m$ are non-degenerate, and that the value of $m$ is constant on the set of all local maximum points [82, Thm. 8.1]. The general case remains open.

It is illustrated here that proper advection can confer some competition advantages. In next subsection, we will use evolutionary game theory to further show that proper advection can be an evolutionary stable strategy. 

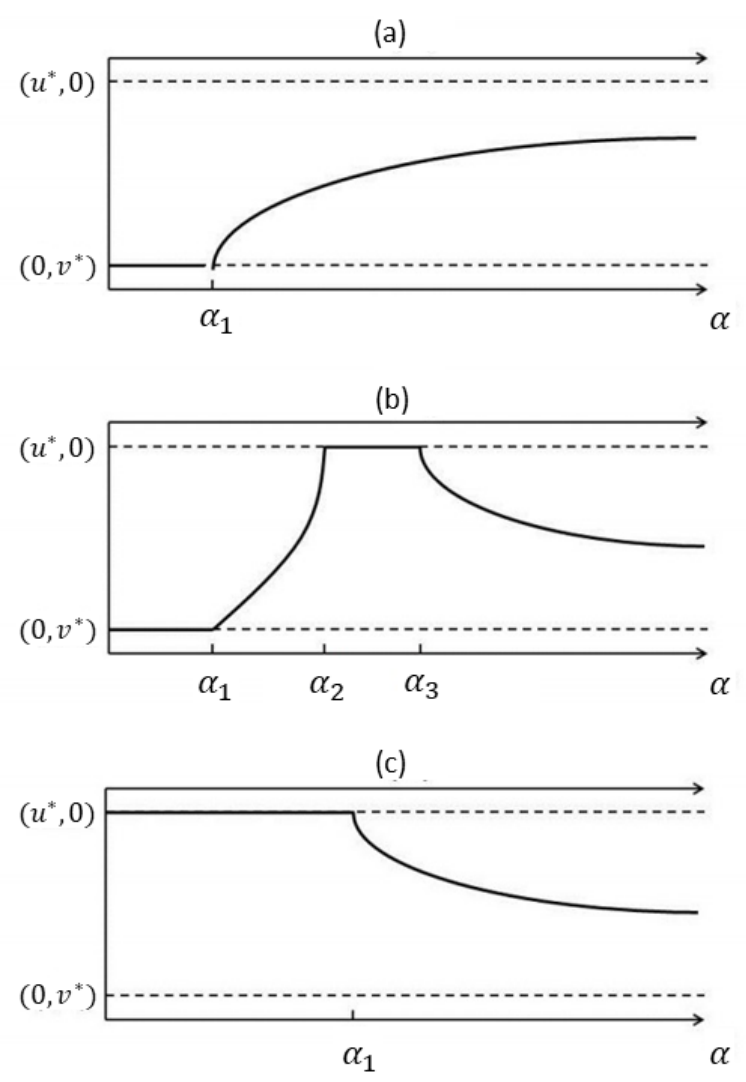

Figure 3. Bifurcation results from [5], where $d, D$ are fixed and $\alpha$ is used as the bifurcation parameter. The three figures, from top to bottom, are the bifurcation diagrams concerning the parameter regions $R_{1}, R_{2}$, and $R_{3}$ (respectively, $D<f(d), f(d)<D<d$ and $D>d$ ) in Figure 2, (First published in Mem. Amer. Math. Soc. 245 (Spring 2017), published by the American Mathematical Society. 2017 American Mathematical Society.)

4.2. Evolution of biased movement. In this subsection, we adopt the viewpoint of adaptive dynamics [36, 54 to consider the evolution of dispersal and the ecological impact of dispersal in spatially heterogeneous environments. An important concept in adaptive dynamics is Evolutionarily Stable Strategy (ESS), known also as Evolutionarily Steady Strategy, which was introduced by Maynard Smith and Price in the seminal paper [119]. A strategy is said to be evolutionarily stable if a population using it cannot be invaded by any small population using a different strategy. ESS is a very general concept. In this subsection we aim to connect it with the local stability of the semi-trivial steady states discussed earlier.

An interesting question is what kind of advection rate will be evolutionarily stable, by regarding the rates of advection along the environmental gradient as movement strategies of 
the species. For this purpose, we consider the following model:

$$
\begin{cases}\partial_{t} u=\nabla \cdot[d \nabla u-\alpha u \nabla m]+u(m-u-v) & (x, t) \in \Omega \times(0, \infty), \\ \partial_{t} v=\nabla \cdot[d \nabla v-\beta v \nabla m]+v(m-u-v) & (x, t) \in \Omega \times(0, \infty), \\ d \frac{\partial u}{\partial \nu}-\alpha u \frac{\partial m}{\partial \nu}=d \frac{\partial v}{\partial \nu}-\beta v \frac{\partial m}{\partial \nu}=0 & (x, t) \in \partial \Omega \times(0, \infty), \\ u(x, 0)=u_{0}(x), v(x, 0)=v_{0}(x) & x \in \Omega,\end{cases}
$$

where $u$ and $v$ are the densities of resident and mutant populations, and the two species differ only in their advection rates, given by the non-negative parameters $\alpha$ and $\beta$, respectively.

The mutant population $v$, which is assumed to be small initially, can invade successfully if and only if the semi-trivial steady state $\left(u^{*}, 0\right)$ is unstable. Here $u^{*}$ denotes the unique positive solution of

$$
\begin{cases}\nabla \cdot[d \nabla u-\alpha u \nabla m]+u(m-u)=0 & x \in \Omega, \\ d \frac{\partial u}{\partial \nu}-\alpha u \frac{\partial m}{\partial \nu}=0 & x \in \partial \Omega .\end{cases}
$$

A natural question is whether there is a critical value $\alpha^{*}>0$ such that $\left(u^{*}, 0\right)$ is a stable steady state of (18) whenever $\alpha=\alpha^{*}$ and $\beta \neq \alpha^{*}$. We call such $\alpha^{*}$, if it exists, an ESS. To find such a magical strategy, we note that the stability of $\left(u^{*}, 0\right)$ is determined by the principal eigenvalue, denoted by $\Lambda_{1}$, of the problem

$$
\begin{cases}\nabla \cdot[d \nabla \varphi-\beta \varphi \nabla m]+\varphi\left(m-u^{*}\right)+\lambda \varphi=0 & x \in \Omega, \\ d \frac{\partial \varphi}{\partial \nu}-\beta \varphi \frac{\partial m}{\partial \nu}=0 & x \in \partial \Omega .\end{cases}
$$

It is classical that the set of eigenvalues of 20 is real and bounded from below. The principal eigenvalue $\Lambda_{1}$ refers to the smallest eigenvalue. It is simple and corresponds to a strictly positive eigenfunction in $\bar{\Omega}$; see [12, 26]. Since $u^{*}$ is an implicit function of $\alpha$, we regard $\Lambda_{1}=\Lambda_{1}(\alpha, \beta)$. Observe that $\Lambda_{1}=0$ for $\alpha=\beta$, since the principal eigenfunction $\varphi$ is simply a constant multiple of $u^{*}$ in such a case.

It is well known that if $\Lambda_{1}>0$, then $\left(u^{*}, 0\right)$ is stable; otherwise, $\left(u^{*}, 0\right)$ is unstable. Hence if $\alpha^{*}$ is an ESS, then $\Lambda_{1}\left(\alpha^{*}, \beta\right) \geq 0$ for all $\beta$, which together with $\Lambda_{1}\left(\alpha^{*}, \alpha^{*}\right)=0$ implies that

$$
\left.\frac{\partial \Lambda_{1}}{\partial \beta}\right|_{\alpha=\beta=\alpha^{*}}=0
$$

We call any strategy $\alpha^{*}$ satisfying (21) an evolutionarily singular strategy, hence any ESS is automatically an evolutionarily singular strategy. For the existence of evolutionarily singular strategies, we have the following result:

Theorem 4.3 ([87, Thm. 2.2]). Suppose that $m \in C^{2}(\bar{\Omega})$ is strictly positive in $\bar{\Omega}$ and $\max _{\bar{\Omega}} m / \min _{\bar{\Omega}} m \leq 3+2 \sqrt{2}$. Given any $A>1 / \min _{\bar{\Omega}} m$, for all small positive $d$, there is exactly one evolutionarily singular strategy $\alpha^{*} \in(0, d A)$.

The condition $\max _{\bar{\Omega}} m / \min _{\bar{\Omega}} m \leq 3+2 \sqrt{2}$ is sharp, and Theorem 4.3 may fail for general $m$ [87, Thm. 2.6]. When $\Omega$ is one-dimensional, the evolutionarily singular strategy found in Theorem 4.3 is also unique in the whole interval $[0, \infty)$, provided $d>0$ is small enough [87, Cor. 2.3]. We conjecture that the uniqueness holds for $\Omega$ of higher dimensions as well. For intermediate or large $d$, however, the uniqueness of singular strategies remains open; see [20] for some recent progress. We conjecture that for any evolutionarily singular strategy 
$\alpha^{*}=\alpha^{*}(d)$, it holds that, for all $d$,

$$
\min _{\bar{\Omega}} m<\frac{d}{\alpha^{*}}<\max _{\bar{\Omega}} m
$$

If this estimate were true, it suggests that a balanced combination of diffusion and advection might be a better movement strategy for populations. The following result shows that this is indeed the case:

Theorem 4.4 ([87, Thm. 2.5]). Suppose that $\Omega$ is convex and $m \in C^{2}(\bar{\Omega})$ is strictly positive in $\bar{\Omega}$. Assume that $\|\nabla(\ln m)\|_{L^{\infty}(\Omega)} \leq \alpha_{0} / \operatorname{diam}(\Omega)$, where $\alpha_{0} \approx 0.8$ and $\operatorname{diam}(\Omega)$ is the diameter of $\Omega$. Then for small $d$, the strategy $\alpha^{*}$ given by Theorem 4.3 is a local ESS, i.e. there exists some small $\delta>0$ such that $\Lambda_{1}\left(\alpha^{*}, \beta\right)>0$ for all $\beta \in\left(\alpha^{*}-\delta, \alpha\right) \cup\left(\alpha, \alpha^{*}+\delta\right)$.

Some other work also considered model $(18)$ and related models. For instance, in [88] we discussed the evolution of diffusion rate in an advective environment. We refer to [20, 24, 25, 28, 29, [53, 55] for further discussions.

Can we decide whether the local ESS $\alpha^{*}$ given in Theorem 4.3 is actually a global ESS? Or more generally, what can we say about the global structure of nodal set for the function $\Lambda_{1}=\Lambda_{1}(\alpha, \beta)$ ? It is clear that the nodal set of $\Lambda_{1}(\alpha, \beta)$ must include the diagonal line $\alpha=\beta$, but otherwise little is known. For the reaction-diffusion models discussed in this paper, determining the complete structure of the nodal set for $\Lambda_{1}(\alpha, \beta)$ poses a challenging problem, both analytically and numerically [52]. To this end, some asymptotic behaviors of $\Lambda_{1}$ were studied in [26, 27, 99, 139] in order to have a better understanding for this problem. Recently, the asymptotic behaviors for the principal eigenvalues of time-periodic parabolic operators were also studied, and we refer to [64, 69, 71, 101, 102, 103, 137. These new developments of the related linear PDE theory may open up a new venue to study the evolution of dispersal in spatio-temporally varying environments.

The evolutionary dynamics, e.g. existence of ESS, have various consequences on the twospecies competition dynamics. This connection has been investigated in [13] for a broad class of model including reaction-diffusion equations and nonlocal diffusion equations. It was shown that frequently the ESS playing species can competitively exclude the species playing a different but nearby strategy, regardless of the initial condition. In the jargon of adaptive dynamics, this result says that ESS implies Neighborhood Invader Strategy. This is quite unexpected, since an ESS by its very definition only concerns the local stability of the semitrivial steady state. The main tool in [13] is Hadamard's graph transform method.

\section{Continuous trait models}

Regarding the advection rates as strategies of the species, in Subsection 4.2 we investigated the course of evolution for two-species competition model (18). Therein the strategies $\{\alpha, \beta\}$ form a discrete set, and the competition and mutation occur independently of each other. In this section, we consider population models with continuous traits, in which the competition and mutation can occur simultaneously.

5.1. Mutation-selection model. To study the selection of random dispersal rate in multispecies competition, Dockery et al. [39] considered the following model:

$$
\partial_{t} u_{i}=\xi_{i} \Delta u_{i}+u_{i}\left[m(x)-\sum_{j=1}^{K} u_{j}\right]+\sum_{j=1}^{K} M_{i j} u_{j},
$$


where $K$ interacting species, with densities $u_{i}(x, t), 1 \leq i \leq K$, compete for a common resource $m(x)$ and differ only in their diffusion rates $\xi_{i}$. These species are subject to mutation with switching rate $M_{i j}$ from phenotype $j$ to $i$. For $K=2$ and $M_{i j}=0$, a well-known result is that the slower diffusing species always win in the competition, but it remains open to determine the global dynamics for the case $K \geq 3$.

Next, we extend the discrete set of strategies $\left\{\xi_{i}\right\}_{i=1}^{K}$ in $(23)$ to a continuum set $(\underline{\xi}, \bar{\xi})$. By viewing the population $u(x, \xi, t)$ as being structured by space, trait and time (i.e. $u(\cdot, \xi, t)$ gives the spatial distribution of the individuals playing strategy $\xi$ at time $t$ ), we obtain the following mutation-selection model:

$$
\begin{cases}\partial_{t} u=\xi \Delta_{x} u+u[m(x)-\hat{u}(x, t)]+\epsilon^{2} \partial_{\xi \xi} u & \text { for } x \in \Omega, \xi \in(\underline{\xi}, \bar{\xi}), t>0, \\ \nu(x) \cdot \nabla_{x} u=0 & \text { for } x \in \partial \Omega, \xi \in(\underline{\xi}, \bar{\xi}), t>0, \\ \partial_{\xi} u=0 & \text { for } x \in \Omega, \xi \in\{\underline{\xi}, \bar{\xi}\}, t>0, \\ u(x, \xi, 0)=u_{0}(x, \xi) & \text { for } x \in \Omega, \xi \in(\underline{\xi}, \bar{\xi}) .\end{cases}
$$

Here the parameters $\underline{\xi}, \bar{\xi}$, which are assumed to be positive, determine the range of continuous strategy $\xi$, and

$$
\hat{u}(x, t):=\int_{\underline{\xi}}^{\bar{\xi}} u\left(x, \xi^{\prime}, t\right) \mathrm{d} \xi^{\prime}
$$

represents the total population density at location $x$ and time $t$, and the term $\epsilon^{2} \partial_{\xi \xi} u$ accounts for the rare mutation, which is modeled by a diffusion with covariance $\sqrt{2} \epsilon$. We refer to 22 for a derivation of this type of equations from individual based on stochastic models. Model (24) is an integro-PDE model describing the diffusion, competition and mutation of a population with continuous traits.

If $\int_{\Omega} m(x) \mathrm{d} x>0$, then it is shown in [85] that 24) admits a unique positive steady state $u_{\epsilon}(x, \xi)$ for small $\epsilon$, which is locally asymptotically stable and satisfies

$$
\begin{cases}\xi \Delta_{x} u+\epsilon^{2} \partial_{\xi \xi} u+u[m(x)-\hat{u}(x)]=0 & (x, \xi) \in \Omega \times(\underline{\xi}, \bar{\xi}), \\ \nu(x) \cdot \nabla_{x} u=0 & (x, \xi) \in \partial \Omega \times(\underline{\xi}, \bar{\xi}), \\ \partial_{\xi} u=0 & (x, \xi) \in \Omega \times\{\underline{\xi}, \bar{\xi}\},\end{cases}
$$

where $\hat{u}(x)=\int_{\underline{\xi}}^{\bar{\xi}} u\left(x, \xi^{\prime}\right) \mathrm{d} \xi^{\prime}$. Observe that when $m$ is constant, i.e. $m \equiv m_{0}$ for some positive constant $m_{0}$, we have $u_{\epsilon} \equiv m_{0} /(\bar{\xi}-\underline{\xi})$ which is globally stable for any $\epsilon>0$ [85]. This suggests that selection does not favor any particular strategy $\xi \in[\underline{\xi}, \bar{\xi}]$, and the mutation $\epsilon^{2} \partial_{\xi \xi} u$ has no effect on the population dynamics in the homogeneous environment. What happens in the spatially heterogeneous environment? The following result gives the answer:

Theorem 5.1 ([86]). Suppose that $\int_{\Omega} m(x) \mathrm{d} x>0$ and $m$ is non-constant. Then as $\epsilon \rightarrow 0$,

$$
\left\|\epsilon^{2 / 3} u_{\epsilon}(x, \xi)-u^{*}(x, \underline{\xi}) \eta^{*}\left(\frac{\xi-\underline{\xi}}{\epsilon^{2 / 3}}\right)\right\|_{L^{\infty}(\Omega \times(\underline{\xi}, \bar{\xi}))} \rightarrow 0,
$$

where $\eta^{*}(s)$ satisfying the following ordinary differential equation:

$$
\left\{\begin{array}{l}
\eta^{\prime \prime}+\left(a_{0}-a_{1} s\right) \eta=0 \quad s>0, \\
\eta^{\prime}(0)=\eta(+\infty)=0, \quad \int_{0}^{\infty} \eta(s) \mathrm{d} s=1,
\end{array}\right.
$$


with some positive constants $a_{0}$ and $a_{1}$. In particular, we have as $\epsilon \rightarrow 0$,

$$
u_{\epsilon}(x, \xi) \rightarrow u^{*}(x, \underline{\xi}) \cdot \delta(\xi-\underline{\xi}) \quad \text { in distribution, }
$$

where $u^{*}(x, \underline{\xi})$ denotes the unique positive solution of (2) with $d=\underline{\xi}$, and $\delta(\cdot)$ is a Dirac mass supported at 0 .

It can be verified that for any $\xi_{0} \in[\underline{\xi}, \bar{\xi}]$, the limit profile $u^{*}\left(x, \xi_{0}\right) \cdot \delta\left(\xi-\xi_{0}\right)$ is a solution of (25) with $\epsilon=0$. Theorem 5.1 says that only the steady state corresponding to the smallest diffusion rate is preserved by the perturbation of $\epsilon=0$ to $0<\epsilon \ll 1$. Theorem 5.1 implies that when the mutation rate is small, the species with the smallest diffusion rate $\underline{\xi}$ will eventually become the dominant phenotype by driving other phenotypes to extinction. This result connects with the case of two-species competition: The smallest available diffusion rate is selected. Note from [85] that the steady state $u_{\epsilon}$ is the unique solution of (25) for small $\epsilon>0$, and it is locally asymptotically stable. An unsolved problem here is the global stability of the unique positive steady state $u_{\epsilon}(x, \xi)$.

A closely related work is due to Perthame and Souganidis [140, where the following mutation-selection model was considered:

$$
\begin{cases}\xi(z) \Delta_{x} u+\epsilon^{2} \partial_{z z} u+u(m(x)-\hat{u})=0 & (x, z) \in \Omega \times[0,1], \\ \nu(x) \cdot \nabla_{x} u=0 & (x, z) \in \partial \Omega \times[0,1], \\ u(x, 0)=u(x, 1) & x \in \Omega,\end{cases}
$$

where $\Omega$ is assumed to be convex and $\xi(z)$ is assumed to be positive and periodic with unit period. For the solution $u_{\epsilon}(x, z)$ of $[26)$, it is proved in [140] that $u_{\epsilon}(x, z) \rightarrow u^{*}\left(x, \xi\left(z_{*}\right)\right)$. $\delta\left(z-\xi\left(z_{*}\right)\right)$ in distribution as $\epsilon \rightarrow 0$, where $\xi\left(z_{*}\right)=\min _{0 \leq z \leq 1} \xi(z)$. This implies that for rare mutations, the population concentrates on a single trait associated to the smallest diffusion rate, in agreement with the result for (25). See also [8, 76, 125] for some progress in this direction.

5.2. Mutation-selection model with drift. Similar to (24), we introduce the following integro-PDE model in a stream:

$$
\begin{cases}\partial_{t} u=\xi \partial_{x x} u-\alpha \partial_{x} u+u[r(x)-\hat{u}(x, t)]+\epsilon^{2} \partial_{\xi \xi} u & \text { for } x \in(0, L), \xi \in(\underline{\xi}, \bar{\xi}), t>0, \\ \left(\xi \partial_{x} u-\alpha u\right)(0, \xi, t)=\left(\xi \partial_{x} u-\alpha u\right)(L, \xi, t)=0 & \text { for } \xi \in(\underline{\xi}, \bar{\xi}), t>0, \\ u(x, \bar{\xi}, t)=u(x, \xi, t)=0 & \text { for } x \in(0, L) t>0, \\ u(x, \xi, 0)=u_{0}(x, \xi) & \text { for } x \in(0, L), \xi \in(\underline{\xi}, \bar{\xi}),\end{cases}
$$

where $\alpha>0$ is the advection rate and $\hat{u}(x, t):=\int_{\underline{\xi}}^{\bar{\xi}} u\left(x, \xi^{\prime}, t\right) \mathrm{d} \xi^{\prime}$ denotes the total population density at location $x$ and time $t$.

When $r$ is a positive constant, from Theorem 3.4 we conjecture that (i) the integro-PDE (27) admits at most one positive steady state, denoted by $u_{\epsilon}(x, \xi)$, which is globally stable whenever it exists; (ii) as $\epsilon \rightarrow 0, u_{\epsilon}(x, \xi) \rightarrow u^{*}(x, \bar{\xi}) \cdot \delta(\xi-\bar{\xi})$ in distribution sense, where $u^{*}(x, \bar{\xi})$ is the unique positive solution of $(14)$ with $D=\bar{\xi}$. Again, this predicts that the larger diffusion is selected as in Theorem 3.4 .

To study the dynamics of (27) for non-constant $r$, we carried out some numerical simulations in [56], where the corresponding parameters were selected as follows:

$$
L=1, \quad \alpha=1, \quad \underline{\xi}=0.5, \quad \bar{\xi}=1.5, \quad r(x)=e^{(1-a) x+a x^{2}}, \quad \epsilon=10^{-3} .
$$



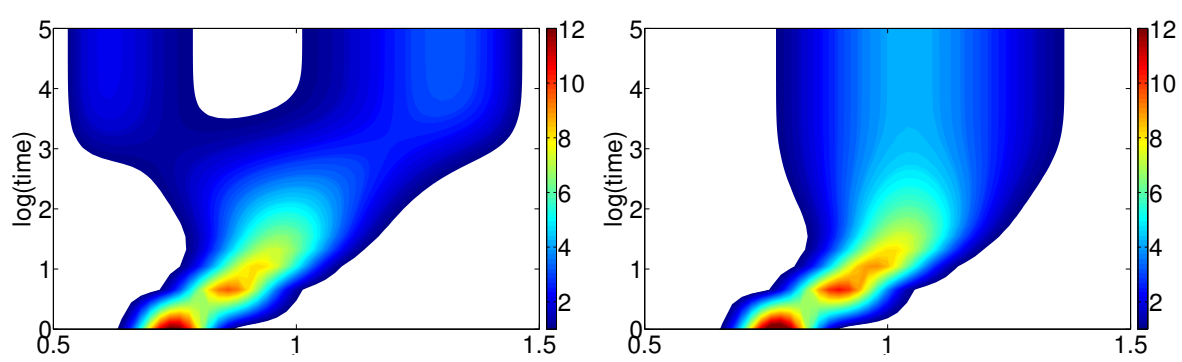

Figure 4. Contour plot of $\int u(x, \xi, t) \mathrm{d} x$ as a function of $\xi$ and time (log(time) for vertical axis) for $a=\frac{1}{4}$ (left) and $a=-\frac{1}{4}$ (right). (First published in [W. Hao et al., Indiana Univ. Math. J. 68 (2019), 881-923.].)

We take initial conditions in the form of one Dirac mass on the phenotypic space, and investigate their evolution for $a= \pm \frac{1}{4}$. The numerical results are presented in Figure 4 .

(i) The numerical result in Figure 4 (right) shows that when $a=\frac{1}{4}$, the steady state of (27) concentrates on the trait $\xi^{*} \approx 1.1$ in the limit of rare mutation, which suggests that the species adopting the strategy $\xi^{*}$ persists and other species will disappear in the competition. This suggests that $\xi^{*}$ is an ESS, which is in contrast with the assertion that "faster diffusion is more favorable" for the homogeneous environment.

(ii) More interestingly, when $a=-\frac{1}{4}$, Figure 4 (left) shows that the steady state of (27) concentrates on two different traits, so that two species adopting different strategies form a coalition that is able to resist invasion by any of the remaining species! This phenomenon is called "evolutionary branching" in adaptive dynamics, and it is also clearly different from the homogeneous case.

To explain these phenomena mathematically, we investigate the asymptotic behaviors of steady state $u_{\epsilon}(x, \xi)$ for (27) as $\epsilon \rightarrow 0$. To this end, we first consider the two-species competition model (13) and denote $\lambda=\lambda\left(\xi_{1}, \xi_{2}\right)$ as the principal eigenvalue of the problem

$$
\begin{cases}\xi_{2} \partial_{x x} \varphi-\alpha \partial_{x} \varphi+\left[r-u^{*}\left(\cdot, \xi_{1}\right)\right] \varphi+\lambda \varphi=0 & x \in(0, L) \\ \xi_{2} \partial_{x} \varphi-\alpha \varphi=0 & x=0, L\end{cases}
$$

where $x \mapsto u^{*}\left(x, \xi_{1}\right)$ is the unique positive solution of (14) with $D=\xi_{1}$. In the adaptive dynamics framework, $\lambda\left(\xi_{1}, \xi_{2}\right)$ is called the invasion fitness, and an invader with trait $\xi_{2}$ can (resp. cannot) invade an established trait $\xi_{1}$ at equilibrium when rare if $\lambda\left(\xi_{1}, \xi_{2}\right)<0$ (resp. $\left.\lambda\left(\xi_{1}, \xi_{2}\right)>0\right)$. The limiting profiles of $u_{\epsilon}(x, \xi)$ depends critically on the behavior of $\lambda\left(\xi_{1}, \xi_{2}\right)$ near $\xi_{2}=\xi_{1}$. Note that $\lambda(\xi, \xi) \equiv 0$ for all $\xi>0$. We first consider the case of $\partial_{\xi_{2}} \lambda(\xi, \xi) \neq 0$, where the following result holds:

Theorem $5.2([56])$. Suppose that $\partial_{\xi_{2}} \lambda(\xi, \xi)>0$ for all $\xi \in(\underline{\xi}, \bar{\xi})$, and $\bar{\xi}-\underline{\xi}$ is sufficiently small. Then any positive steady state $u_{\epsilon}$ of 27) satisfies as $\epsilon \rightarrow 0$,

$$
u_{\epsilon}(x, \xi) \rightarrow \delta(\xi-\underline{\xi}) \cdot u^{*}(x, \underline{\xi}) \quad \text { in distribution, }
$$

where $x \mapsto u^{*}(x, \underline{\xi})$ is the unique positive solution of (14) with $D=\underline{\xi}$.

Theorem 5.2 implies that for the case $\partial_{\xi_{2}} \lambda(\xi, \xi)>0$, the slowest diffusion will be selected in the competition. Similarly, we may conclude that if $\partial_{\xi_{2}} \lambda(\xi, \xi)<0$ for all $\xi \in(\underline{\xi}, \bar{\xi})$, then the steady state $u_{\epsilon}$ concentrates on the trait $\xi=\bar{\xi}$, so that the fastest diffusion will be selected, coinciding with the observation in Theorem 3.4 . These results show that if $\partial_{\xi_{2}} \lambda \neq 0$, it gives 
rise to a single Dirac-concentration at one of the two extreme traits, depending upon the sign of $\partial_{\xi_{2}} \lambda$.

To study the case when $\partial_{\xi_{2}} \lambda$ vanishes somewhere in $(\underline{\xi}, \bar{\xi})$, we introduce the convergence stable strategy $\hat{\xi}$ (see [45]), which is characterized by the following relations:

$$
\partial_{\xi_{2}} \lambda(\hat{\xi}, \hat{\xi})=0 \quad \text { and }\left.\quad \frac{\mathrm{d}}{\mathrm{ds}}\left[\partial_{\xi_{2}} \lambda(s, s)\right]\right|_{s=\hat{\xi}}>0 .
$$

This leads to two generic cases: The convergence stable strategy $\hat{\xi}$ is an ESS if $\partial_{\xi_{2}}^{2} \lambda(\hat{\xi}, \hat{\xi})>0$, and is a branching point $(\mathrm{BP})$ if $\partial_{\xi_{2}}^{2} \lambda(\hat{\xi}, \hat{\xi})<0$.

In the case when the ESS exists, there is concentration in the mutation-selection model.

Theorem 5.3. [56] Suppose that $\hat{\xi}$ is an ESS and (29) holds. If $\hat{\xi} \in(\underline{\xi}, \bar{\xi})$ and $\bar{\xi}-\underline{\xi}$ is sufficiently small, then as $\epsilon \rightarrow 0$, any positive steady state $u_{\epsilon}$ of (27) satisfies

$$
u_{\epsilon}(x, \xi) \rightarrow u^{*}(x, \hat{\xi}) \cdot \delta(\xi-\hat{\xi}) \quad \text { in distribution. }
$$

Theorem 5.3 indicates that if there is an ESS in $(\xi, \bar{\xi})$, then the phenotype adopting the ESS dominates the competition in the limit of rare mutation. This corresponds to the numerical result in Figure 4 (right) with $\hat{\xi} \approx 1.1$. To further understand Theorem 5.3 , we consider a special case of (13) where $r(x)=b e^{c x}$ for some positive constants $b$ and $c$. In this case, it is shown in [4] that the semi-trivial state $\left(u^{*}\left(x, \xi_{1}\right), 0\right)$ is globally stable when $\xi_{1}=\alpha / c$ and $\xi_{2} \neq \alpha / c$, i.e. the intermediate trait $\hat{\xi}=\alpha / c$ is an ESS. We can offer some intuitive reasoning for this result by employing the theory of ideal free distribution [46]: When $r(x)=b e^{c x}$, if $\xi_{1}=\alpha / c$, it can be verified from (14) that $u^{*}\left(x, \xi_{1}\right) \equiv b e^{c x} \equiv r(x)$, so that species $u$ attains the ideal free distribution, and thus $\alpha / c$ being an ESS is natural. As $c \rightarrow 0$, i.e. the environment tends to be homogeneous, we find $\hat{\xi}=\alpha / c$ approaches $+\infty$, which is in agreement with the prediction of Theorem 3.4 .

Finally, in the neighborhood of a BP, we have the following result:

Theorem 5.4 ([56]). Suppose that $\hat{\xi} \in(\underline{\xi}, \bar{\xi})$ is a $\mathrm{BP}$ and $(29)$ holds. If $\bar{\xi}-\underline{\xi}$ is sufficiently small, then as $\epsilon \rightarrow 0$, any positive steady state $u_{\epsilon}$ of (27) satisfies

$$
u_{\epsilon}(x, \xi) \rightarrow \delta(\xi-\underline{\xi}) \cdot u_{1}(x)+\delta(\xi-\bar{\xi}) \cdot u_{2}(x) \quad \text { in distribution, }
$$

where $\left(u_{1}, u_{2}\right)$ is a positive solution of

$$
\begin{cases}\underline{\xi} \partial_{x x} u_{1}-\alpha \partial_{x} u_{1}+u_{1}\left(r-u_{1}-u_{2}\right)=0 & x \in(0, L), \\ \bar{\xi} \partial_{x x} u_{2}-\alpha \partial_{x} u_{2}+u_{2}\left(r-u_{1}-u_{2}\right)=0 & x \in(0, L), \\ \underline{\xi} \partial_{x} u_{1}-\alpha u_{1}=\bar{\xi} \partial_{x} u_{2}-\alpha u_{2}=0 & x=0, L .\end{cases}
$$

Theorem 5.4 reveals a new phenomenon: In the neighborhood of a BP, no single trait can dominate the competition; instead, the two extreme traits, $\xi$ and $\bar{\xi}$, form a coalition that together dominates the competition such that any species with other traits cannot invade. To illustrate Theorem 5.4, we consider the case $r(x)=b_{1} e^{c_{1} x}+b_{2} e^{c_{2} x}$ in (13) with positive constants $b_{i}$ and $c_{i}, i=1,2$. We observe that if $\xi=\alpha / c_{1}$ and $\bar{\xi}=\alpha / c_{2}$, then (30) has the unique positive solution $u_{i}(x)=b_{i} e^{c_{i} x}, i=1,2$. Again, in the framework of ideal free distribution, it is not difficult to understand the biological reasoning: In view of $u_{1}(x)+$ $u_{2}(x)=b_{1} e^{c_{1} x}+b_{2} e^{c_{2} x}=r(x)$, the total density of the two species can jointly reach an ideal free distribution, so that $\underline{\xi}=\alpha / c_{1}$ and $\bar{\xi}=\alpha / c_{2}$ potentially become a pair of ESS. We refer to [17, 18, 19, 21, 53] for related work on the evolution of dispersal and ideal free distribution. 
An open problem is whether (27) or (30) has at most one positive solution, and if it exists, whether it is globally asymptotically stable. Another challenging question is to determine the limit of the time-dependent solutions $u_{\varepsilon}(x, \xi, t)$ of (25) and (27) as $\epsilon \rightarrow 0$. See [94, 132] and references therein for recent progress on the rigorous derivations of the canonical equations for the trait evolution in spatially structured mutation-selection models.

\section{Dynamics of phytoplankton}

Besides the models discussed above, there are many other types of reaction-diffusion models in population dynamics. In this section, we briefly discuss some problems arising from the phytoplankton growth.

Phytoplankton are microscopic plant-like photosynthetic organisms drifting in lakes and oceans and are the foundation of the marine food chain. Since they transport significant amounts of atmospheric carbon dioxide into the deep oceans, they play a crucial role in climate dynamics and have been one of the central topics in marine ecology. Nutrients and light are the essential resources for the growth of phytoplankton. Since most phytoplankton are heavier than water, they will sink into the bottom of the lakes or oceans where the light intensity is too weak for their growth. So how do these phytoplankton persist in the water columns? Some biologists propose that biased movement, combining with water turbulence, can help phytoplankton diffuse to a position closer to the top of lakes or oceans, so that they can get access to sunlight. In this connection, a series of reaction-diffusion models including single and multiple phytoplankton species are introduced in [66, 67, 68] and the references therein to model the spatio-temporal dynamics of phytoplankton growth.

The following system of reaction-diffusion-advection equations was used by Huisman et al. [68. to describe the population dynamics of two phytoplankton species:

$$
\left\{\begin{array}{lc}
\partial_{t} u=D_{1} \partial_{x x} u-\alpha_{1} u_{x}+\left[g_{1}(I(x, t))-d_{1}\right] u & 0<x<L, t>0, \\
\partial_{t} v=D_{2} \partial_{x x} v-\alpha_{2} v_{x}+\left[g_{2}(I(x, t))-d_{2}\right] v & 0<x<L, t>0, \\
D_{1} \partial_{x} u(x, t)-\alpha_{1} u(x, t)=0 & x=0, L, t>0, \\
D_{2} \partial_{x} v(x, t)-\alpha_{2} v(x, t)=0 & x=0, L, t>0, \\
u(x, 0)=u_{0}(x) \geq, \not \equiv 0, \quad v(x, 0)=v_{0}(x) \geq, \not \equiv 0 & 0 \leq x \leq L .
\end{array}\right.
$$

Here $u(x, t), v(x, t)$ denote the population density of the phytoplankton species at depth $x$ and time $t$, and $D_{1}, D_{2}>0$ are their diffusion rates. For $i=1,2, \alpha_{i} \in \mathbb{R}$ is the sinking $\left(\alpha_{i}>0\right)$ or buoyant $\left(\alpha_{i}<0\right)$ velocity, $d_{i}>0$ is the death rate, and $g_{i}(I)$ represents the specific growth rate of phytoplankton species as a function of light intensity $I$, given by the Lambert-Beer law

$$
I(x, t)=I_{0} \exp \left[-k_{0} x-\int_{0}^{x}\left(k_{1} u(s, t)+k_{2} v(s, t)\right) \mathrm{d} s\right],
$$

where $I_{0}>0$ is the incident light intensity, $k_{0}>0$ is the background turbidity that summarizes light absorption by all non-phytoplankton components, and $k_{i}$ is the absorption coefficient of the $i$-th phytoplankton species. Function $g_{i}(I)$ is smooth and satisfies

$$
g_{i}(0)=0 \text { and } g_{i}^{\prime}(I)>0 \text { for } I \geq 0 .
$$

6.1. Single phytoplankton species. The single species model, i.e. $v=0$ in (31), was first considered in [141] for the self-shading case and infinite long water column; see also [75, 81. The authors 44] considered the global dynamics of the single species model when diffusion 
and drift rates are spatially dependent. In [42] the authors studied the effect of photoinhibition on the single phytoplankton species, and they found that, in contrast to the case of no photoinhibition, the model with photoinhibition possesses at least two positive steady states in certain parameter ranges. Hsu et al. [73] studied the single species growth consuming inorganic carbon with internal storage in a poorly mixed habitat, building upon some interesting recent findings of the principal eigenvalue of a 1-homogeneous positive compact operator. In [137, 138, the authors considered the effect of time-periodic light intensity at the surface. $\mathrm{Ma}$ and $\mathrm{Ou}$ [118] recently made an important finding that the biomass of the single species satisfies a comparison principle, even though the density itself does not obey such orders.

In [72, we investigated a single phytoplankton model and obtained some necessary and sufficient conditions for the growth of phytoplankton, and the critical death rate, critical water column depth, critical sinking or buoyant coefficient and critical turbulent diffusion rate were studied respectively. One of the results is that the phytoplankton population persists if and only if the sinking velocity of phytoplankton is less than a critical value. There are many simplified assumptions in the model, e.g. the death rate is assumed to be a constant. An interesting issue is to consider the case when the death rate is non-constant in space and time. Some preliminary results show that this situation is quite different from that of constant death rate. For instance, under some conditions phytoplankton can persist if and only if the sinking velocity stays within an intermediate range, rather than below a single critical value.

6.2. Two phytoplankton species. The coexistence of two or multiple phytoplankton is an important issue. In contrast to single phytoplakton species, very few results exist for two or more phytoplankton species; see [40, 41, 124]. On the one hand, the coexistence of many species can often be observed in reality. On the other hand, the classical competition theory shows that only the most dominant phytoplankton persists. They seem to contradict each other. The reason is that the classical competition theory is generally established for ordinary differential equation models, i.e. it is assumed that the diffusion rates of phytoplankton are sufficiently large, so that only their average densities are considered; i.e. the water column is well mixed. A natural question is whether small diffusion can increase the possibility of coexistence. In this connection, we recently investigated the outcome of model (31)-(32) in [77] and established the following results:

(i) If two phytoplankton differ only in their sinking velocities $\left(D_{1}=D_{2}, \alpha_{1} \neq \alpha_{2}, g_{1}=g_{2}\right.$, $d_{1}=d_{2}$ ), in [77] we showed that the phytoplankton with smaller sinking velocity has the competitive advantage. In terms of evolution, the mutation of phytoplankton can continually reduce their sinking velocities, so that the density of phytoplankton and water will get closer as the population evolves. In other words, as the environment permits, the natural selection may favor the phytoplankton whose density is lighter that of water in some circumstances.

(ii) If two phytoplankton differ only in their diffusion rates $\left(D_{1} \neq D_{2}, \alpha_{1}=\alpha_{2}, g_{1}=\right.$ $\left.g_{2}, d_{1}=d_{2}\right)$, it is shown in [77] that slower diffusion rate will be selected when buoyant, and in contrast, faster diffuser wins when they are sinking with large velocity. An underlying biological reasoning is that when the phytoplankton are buoyant, the slower diffuser are more likely to reach the top of the water column (i.e. without water turbulence), where the light intensity is the strongest, while when sinking with large velocity, faster diffusion can counterbalance the tendency to sink and provide individuals with better access to light.

An important tool is the extension of the comparison principle in [118] for single-species models to two-species phytoplakton models as follows: 
Theorem 6.1. Suppose $\left\{\left(u_{i}, v_{i}\right)\right\}_{i=1,2}$ are non-negative solutions of (31)-(32) such that

$$
\int_{0}^{x} u_{1}(s, 0) \mathrm{d} s \leq, \not \equiv \int_{0}^{x} u_{2}(s, 0) \mathrm{d} s \quad \text { and } \quad \int_{0}^{x} v_{1}(s, 0) \mathrm{d} s \geq, \not \equiv \int_{0}^{x} v_{2}(s, 0) \mathrm{d} s
$$

hold for all $x \in(0, L]$. Then for all $x \in(0, L]$ and $t>0$,

$$
\int_{0}^{x} u_{1}(s, t) \mathrm{d} s<\int_{0}^{x} u_{2}(s, t) \mathrm{d} s \quad \text { and } \quad \int_{0}^{x} v_{1}(s, t) \mathrm{d} s>\int_{0}^{x} v_{2}(s, t) \mathrm{d} s .
$$

By Theorem 6.1, system (31)- 32 is a strongly monotone dynamical system with respect to the order generated by the cone $\mathcal{K}:=\mathcal{K}_{1} \times\left(-\mathcal{K}_{1}\right)$, where

$$
\mathcal{K}_{1}:=\left\{\phi \in C([0, L] ; \mathbb{R}): \int_{0}^{x} \phi(s) \mathrm{d} s \geq 0, \forall x \in(0, L]\right\} .
$$

This in turn enables the application of the theory of strongly monotone dynamical system, which provides a powerful tool to investigate the global dynamics of two-species system (31)(32).

The above findings in part (ii) naturally lead to the following conjecture:

Conjecture 4. Suppose that $\alpha_{1}=\alpha_{2}:=\alpha, g_{1}=g_{2}$ and $d_{1}=d_{2}$. There exist two critical sinking velocities $\alpha_{\min }$ and $\alpha_{\max }$ such that the slower diffuser wins if $\alpha<\alpha_{\min }$, the faster diffuser wins if $\alpha>\alpha_{\max }$, and two species coexist if $\alpha \in\left(\alpha_{\min }, \alpha_{\max }\right)$.

\section{Spatial dynamics of epidemic diseases}

The COVID-19 pandemic has impacted or changed almost everyone's life. There are many mysteries about the novel coronavirus, among which the effect and possible control strategies of the movement of individuals is an emerging question. The COVID-19 pandemic has been spreading so fast, at least partially due to the movement of those infected individuals who are showing little or no symptoms. What is the general impact of movement on the persistence and extinction of a disease in spatially heterogeneous environment? We studied various susceptible-infected-susceptible (SIS) models in the heterogeneous environment [1, 2, 3], including the following reaction-diffusion model [2], where the susceptible and infected individuals move randomly and the disease transmission and recovery rates could be uneven across the space:

$$
\begin{cases}\partial_{t} S-d_{S} \Delta S=-\frac{\beta S I}{S+I}+\gamma I & (x, t) \in \Omega \times(0, \infty), \\ \partial_{t} I-d_{I} \Delta I=\frac{\beta S I}{S+I}-\gamma I & (x, t) \in \Omega \times(0, \infty), \\ \frac{\partial S}{\partial \nu}=\frac{\partial I}{\partial \nu}=0 & (x, t) \in \partial \Omega \times(0, \infty), \\ S(x, 0)=S_{0}(x), I(x, 0)=I_{0}(x) & x \in \Omega,\end{cases}
$$

where $\Omega$ is a bounded domain in $\mathbb{R}^{N}$ with smooth boundary $\partial \Omega$ and $\nu$ denotes the unit outward normal vector on $\partial \Omega$. Here $S(x, t)$ and $I(x, t)$ represent the density of susceptible and infected populations at location $x$ and time $t$, respectively. Parameters $d_{S}, d_{I}>0$ denote their diffusion rates. The functions $\beta(x), \gamma(x)$ account for the rates of disease transmission and recovery, respectively. They are assumed to be positive and at least one of them is non-constant to reflect the spatial heterogeneity of the residing habitat.

In [2], we defined the basic reproduction number $\mathcal{R}_{0}$ for the spatial SIS model (33), which is consistent with the next generation approach for heterogeneous populations [37, 146, 150]. It 
is of practical significance to consider the effect of spatial heterogeneity of disease transmission and recovery rates on the basic reproduction number. For instance, in the study of dengue fever, it is shown in [149] that the infection risk may be underestimated if the spatially averaged parameters are used to compute the basic reproduction number for spatially heterogeneous infections. We proved that $\mathcal{R}_{0}$ is monotone decreasing with respect to the diffusion rate of the infected individuals. The basic reproduction number $\mathcal{R}_{0}$ characterizes the infection risk of disease and serves as the threshold value for the extinction and persistence of disease: Namely, if $\mathcal{R}_{0}<1$, the unique disease-free equilibrium is globally stable, and if $\mathcal{R}_{0}>1$, the disease-free equilibrium is unstable and there is a unique endemic equilibrium. A standing open question is whether this unique endemic equilibrium is globally stable.

Interestingly, we also found in [2] that a disease may persist but it can be controlled by limiting the movement of the susceptible populations. To be more specific, if the spatial environment can be transformed to include low-risk sites where the recovery rates are greater than transmission rates (such as through vaccination and treatment), and the movement of the susceptible individuals can be restricted (such as through isolation or lock down), then it may be possible to control the disease by flattening the disease outbreak curve, i.e. decreasing the daily number of infected individuals.

In recent years there have been many studies on SIS and other types of disease transmission models in spatially and/or temporally varying environments; see [31, 32, 33, 34, 48, 49, 50, 51, 95, 96, 120, 133, 134, 135, 136, 154. For instance, to study the effect of the movement of exposed individuals on disease outbreaks, the following SEIRS (susceptible-exposed-infectedrecovered-susceptible) epidemic reaction-diffusion model was considered in [143]:

$$
\begin{cases}\partial_{t} S=d_{S} \Delta S-\frac{\beta(x) S I}{S+I+E+R}+\alpha R & (x, t) \in \Omega \times(0, \infty), \\ \partial_{t} E=d_{E} \Delta E+\frac{\beta(x) S I}{S+I+E+R}-\sigma E & (x, t) \in \Omega \times(0, \infty), \\ \partial_{t} I=d_{I} \Delta I+\sigma E-\gamma(x) I & (x, t) \in \Omega \times(0, \infty), \\ \partial_{t} R=d_{R} \Delta R+\gamma(x) I-\alpha R & (x, t) \in \Omega \times(0, \infty), \\ \frac{\partial S}{\partial \nu}=\frac{\partial E}{\partial \nu}=\frac{\partial I}{\partial \nu}=\frac{\partial R}{\partial \nu}=0 & (x, t) \in \partial \Omega \times(0, \infty) .\end{cases}
$$

Here we divide the individuals into four different compartments: susceptible $(S)$, exposed $(E)$, infectious $(I)$, recovered (immune by vaccination, $R$ ). The susceptible individuals are infected by infectious individuals with a rate of $\beta$, and become exposed; exposed individuals become infectious with a rate $\sigma$; infected individuals are recovered with a rate $\gamma$; recovered individuals lose immunity and go back into the susceptible class with a rate of $\alpha$. Here $S(x, t)$, $E(x, t), I(x, t)$ and $R(x, t)$ denote the density of susceptible, exposed, infected and recovered individuals at location $x$ and time $t$, and $d_{S}, d_{E}, d_{I}, d_{R}$ represent the diffusion rates for susceptible, exposed, infected and recovered populations, respectively. We assume that the disease transmission rate $\beta(x)$ and recovery rate $\gamma(x)$ are environmentally dependent and could be spatially heterogeneous. Our results in [143] reveal that the travel of exposed individuals could have an important impact on the persistence of disease and the movement of recovered individuals may enhance the endemic. Hence, further understanding of the behaviors of the exposed and recovered individuals could be important in designing effective disease control measures.

In [100] we investigated the spatial SIS model (33) with spatially heterogeneous and timeperiodic coefficients and proved that the basic reproduction number $\mathcal{R}_{0}$ is non-decreasing 
with respect to the time-period $T$. In some scenario, this would imply that there is a critical period $T^{*}>0$ such that $\mathcal{R}_{0}<1$ for $T<T^{*}$ and $\mathcal{R}_{0}>1$ for $T>T^{*}$; i.e. increasing the period of disease transmissions and recovery will increase the chance of disease outbreak. This might suggest why the outbreak of some epidemic diseases are less frequent than others, e.g. annually vs bi-annually, as different epidemic diseases could have different transmission and recovery rates so that the threshold values $T^{*}$ are different.

How do movement and spatial heterogeneity affect the competition among multiple strains? Many diseases are present in multiple phenotypes or strains, e.g. the novel coronavirus has found three major strains which are prevalent in different continents. It is an important evolutionary problem to determine the environmental conditions under which the adaptability of a certain strain becomes dominant. Bremermann and Thieme [9] showed that for an SIR model with one host and multiple pathogens, different pathogens will die out eventually except those that optimize the basic reproduction number. Recent studies showed that for some non-autonomous multi-strain models, even if the transmission and recovery rates change timeperiodically, these strains cannot coexist; i.e. the temporal heterogeneity may not increase the chance of the coexistence for multiple pathogens. Tuncer and Martcheva [145] and Wu et al. 153. considered the following two-strain SIS spatial model to address the question whether the presence of spatial structure would allow two strains to coexist, as the corresponding spatially homogeneous model generally leads to competitive exclusion:

$$
\begin{cases}\partial_{t} S-d_{S} \Delta S=-\frac{S\left(\beta_{1} I_{1}+\beta_{2} I_{2}\right)}{S+I_{1}+I_{2}}+\gamma_{1} I_{1}+\gamma_{2} I_{2} & (x, t) \in \Omega \times(0, \infty), \\ \partial_{t} I_{i}-d_{I, i} \Delta I_{i}=\frac{\beta_{i} S I_{i}}{S+I_{1}+I_{2}}-\gamma_{i} I_{i} & (x, t) \in \Omega \times(0, \infty), \\ \frac{\partial S}{\partial \nu}=\frac{\partial I_{i}}{\partial \nu}=0 & (x, t) \in \partial \Omega \times(0, \infty), \\ S(x, 0)=S_{0}(x), I_{i}(x, 0)=I_{0, i}(x) & x \in \Omega,\end{cases}
$$

where $i=1,2$. These two strains could be different in dispersal rates, transmission rates and recovery rates. For the two pathogens differing only in their diffusion rates, a conjecture is that the pathogen with the slower diffusion will drive the other one to extinction for proper transmission and recovery rates; see [153] for some partial results. For the two pathogens differing only in their recovery rates, we conjecture that the strain with the recovery rate of greater spatial variation will be selected eventually. The coexistence of multiple pathogens in spatially heterogeneous and temporally varying environment remains a promising open research direction.

Acknowledgment. We thank the anonymous referee and Grégoire Nadin and Lei Zhang for their helpful comments. KYL and YL are partially supported by the NSF grant DMS-1853561; SL was partially supported by the Outstanding Innovative Talents Cultivation Funded Programs 2018 of Renmin Univertity of China and the NSFC grant No. 11571364.

\section{REFERENCES}

[1] L. Allen, B. Bolker, Y. Lou, A. Nevai, Asymptotic profile of the steady states for an SIS epidemic patch model, SIAM J. Appl. Math. 67 (2007), 1283-1309.

[2] L. Allen, B. Bolker, Y. Lou, A. Nevai, Asymptotic profile of the steady states for a spatial SIS epidemic disease reaction-diffusion model, Discrete Contin. Dyn. Syst. 21 (2008), 145-164. 
[3] L. Allen, Y. Lou, A. Nevai, Spatial patterns in a discrete-time SIS patch model, J. Math Biol. 58 (2009), 339-375.

[4] I. Averill, Y. Lou, D. Munther, On several conjectures from evolution of dispersal, J. Biol. Dyn. 6 (2012), 117-130.

[5] I. Averill, K.-Y. Lam, Y. Lou, The role of advection in a two-species competition model: a bifurcation approach, Mem. Amer. Math. Soc. 245 (2017): 1161.

[6] X. Bai, X. He, F. Li, An optimization problem and its application in population dynamics, Proc. Amer. Math. Soc. 144 (2016), 2161-2170.

[7] F. Belgacem, C. Cosner, The effects of dispersal along environmental gradients on the dynamics of populations in heterogeneous environment, Canadian Appl. Math. Quarterly 3 (1995), 379-397.

[8] E. Bouin, S. Mirrahimi, A Hamilton-Jacobi limit for a model of population structured by space and trait, Comm. Math Sci. 13 (2015), 1431-1452.

[9] H. Bremermann, H.Thieme, A competitive exclusion principle for pathogen virulence, J. Math. Biol. 27 (1989), 179-190.

[10] P.N. Brown, Decay to uniform states in ecological interactions, SIAM J. Appl. Math. 38 (1980), 22-37.

[11] F. Caubet, T. Deheuvels, Y. Privat, Optimal location of resources for biased movement of species: The $1 D$ Case, SIAM J. Appl. Math. 77 (2017), 1876-1903.

[12] R.S. Cantrell, C. Cosner, Spatial Ecology via reaction-diffusion Equations, Series in Mathematical and Computational Biology, John Wiley and Sons, Chichester, UK, 2003.

[13] R.S. Cantrell, C. Cosner, K-Y. Lam, On resident-invader dynamics in infinite dimensional dynamical systems, J. Differential Equations 263 (2017), 4565-4616.

[14] R.S. Cantrell, C. Cosner, Y. Lou, Multiple reversals of competitive dominance in ecological reserves via external habitat degradation, J. Dyn. Diff. Eqs. 16 (2004), 973-1010.

[15] R.S. Cantrell, C. Cosner, Y. Lou, Movement towards better environments and the evolution of rapid diffusion, Math. Biosci. 240 (2006), 199-214.

[16] R.S. Cantrell, C. Cosner, Y. Lou, Advection-mediated coexistence of competing species, Proc. Roy. Soc. Edinburgh Sect. A 137 (2007), 497-518.

[17] R.S. Cantrell, C. Cosner, Y. Lou, Evolution of dispersal and the ideal free distribution, Math. Biosci. Eng. 7 (2010), 17-36.

[18] R.S. Cantrell, C. Cosner, Y. Lou, Evolutionary stability of ideal free dispersal strategies in patchy environments, J. Math. Biol. 65 (2012), 943-965.

[19] R.S. Cantrell, C. Cosner, Y. Lou, D. Ryan, Evolutionary stability of ideal free dispersal strategies: a nonlocal dispersal model, Can. Appl. Math. Q. 20 (2012), 15-38.

[20] R.S. Cantrell, C. Cosner, M.A. Lewis, Y. Lou, Evolution of dispersal in spatial population models with multiple timescales, J. Math. Biol. 80 (2020), 3-37.

[21] R.S. Cantrell, C. Cosner, Y. Lou, S.J. Schreiber, Evolution of natal dispersal in spatially heterogenous environments, Math. Biosci. 283 (2017), 136-144.

[22] N. Champagnat, R. Ferrière, S. Méléard, Unifying evolutionary dynamics: From individual stochastic processes to macroscopic models, Theor. Pop. Biol. 69 (2006), 297-321.

[23] S.S. Chen, J.P. Shi, Global dynamics of the diffusive Lotka-Volterra competition model with stage structure, Calc. Var. Partial Differential Equations 59 (2020): 33.

[24] X.F. Chen, R. Hambrock, Y. Lou, Evolution of conditional dispersal, a reaction-diffusion-advection model, J. Math. Biol. 57 (2008), 361-386.

[25] X.F. Chen, K.-Y. Lam, Y. Lou, Dynamics of a reaction-diffusion-advection model for two competing species, Discrete Contin. Dyn. Syst. 32 (2012), 3841-3859.

[26] X.F. Chen, Y. Lou, Principal eigenvalue and eigenfunctions of an elliptic operator with large advection and its application to a competition model, Indiana Univ. Math. J. 57 (2008), 627-657. 
[27] X.F. Chen, Y. Lou, Effects of diffusion and advection on the smallest eigenvalue of an elliptic operators and their applications, Indiana Univ. Math J. 60 (2012), 45-80.

[28] C. Cosner, Beyond Diffusion: Conditional Dispersal in Ecological Models, Infinite dimensional dynamical systems, 305-317, Fields Inst. Commun. 64, Springer, New York, 2013.

[29] C. Cosner, Reaction-diffusion-advection models for the effects and evolution of dispersal, Discrete Contin. Dyn. Syst. 34 (2014), 1701-1745.

[30] C. Cosner, Y. Lou, Does movement toward better environments always benefit a population?, J. Math. Anal. Appl. 277 (2003), 489-503.

[31] R. Cui, K.-Y. Lam, Y. Lou, Dynamics and asymptotic profiles of steady states of an epidemic model in advective environments, J. Differential Equations 263 (2017), 2343-2373.

[32] R. Cui, Y. Lou, A spatial SIS model in advective heterogeneous environments, J. Differential Equations 261 (2016), 3305-3343.

[33] K. Deng, Asymptotic behavior of an SIR reaction-diffusion model with a linear source, Discrete Contin. Dyn. Syst. Ser. B 24 (2019), 5945-5957.

[34] K. Deng, Y. Wu, Dynamics of a susceptible-infected-susceptible epidemic reaction-diffusion model, Proc. Roy. Soc. Edinburgh Sect. A 146 (2016), 929-946.

[35] D. DeAngelis, W.-M. Ni, B. Zhang, Dispersal and spatial heterogeneity: single species, J. Math. Biol. 72 (2016), 239-254.

[36] O. Diekmann, A beginners guide to adaptive dynamics, in: Mathematical modelling of population dynamics, Banach Center Publications, Vol. 63, Institute of Mathematics Polish Academy of Sciences, Warszawa, 2004, 47-86.

[37] O. Diekmann, J.A.P. Heesterbeek, J.A.J. Metz, On the definition and the computation of the basic reproduction ratio R0 in models for infectious diseases in heterogeneous populations, J. Math. Biol. 28 (1990), 365-382.

[38] W. Ding, H. Finotti, S. Lenhart, Y. Lou, Q. Ye, Optimal control of growth coefficient on a steady-state population model, Nonlinear Anal. Real World Appl. 11 (2010), 688-704.

[39] J. Dockery, V. Hutson, K. Mischaikow, M. Pernarowski, The evolution of slow dispersal rates: a reactiondiffusion model, J. Math. Biol. 37 (1998), 61-83.

[40] Y. Du, S.-B. Hsu, Concentration phenomena in a nonlocal quasi-linear problem modeling phytoplankton I: existence, SIAM J. Math. Anal. 40 (2008), 1419-1440.

[41] Y. Du, S.-B. Hsu, Concentration phenomena in a nonlocal quasi-linear problem modeling phytoplankton II: limiting profile, SIAM J. Math. Anal. 40 (2008), 1441-1470.

[42] Y. Du, S.-B. Hsu, Y. Lou, Multiple steady-states in phytoplankton population induced by photoinhibition, J. Differential Equations 258 (2015), 2408-2434.

[43] Y. Du, B. Lou, R. Peng, M. Zhou, The Fisher-KPP equation over simple graphs: Varied persistence states in river networks, J. Math. Biol. 80 (2020), 1559-1616.

[44] Y. Du, L. Mei, On a nonlocal reaction-diffusion-advection equation modeling phytoplankton dynamics, Nonlinearity 24 (2011), 319-349.

[45] I. Eshel, U. Motro, Kin selection and strong evolutionary stability of mutual help, Theor. Pop. Biol. 19 (1981), 420-433.

[46] S.D. Fretwell, H.L. Lucas, On territorial behavior and other factors influencing habitat selection in birds, Acta Biotheretica 19 (1970), 16-36.

[47] A. Friedman, What is mathematical biology and how useful is it? Notices Amer. Math. Soc. 57 (2010), 851-857.

[48] D. Gao, Travel frequency and infectious disease, SIAM J. Appl. Math. 79 (2019), 1581-1606.

[49] D. Gao, C. Dong, Fast diffusion inhibits disease outbreaks, Proc. Amer. Math. Soc. 148 (2020), 1709-1722. 
[50] D. Gao, S. Ruan, An SIS patch model with variable transmission coefficients, Math. Biosci. 232 (2011), 110-115.

[51] J. Ge, K.I. Kim, Z. Lin, H. Zhu, An SIS reaction-diffusion-advection model in a low-risk and high-risk domain, J. Differential Equations 259 (2015), 5486-5509.

[52] M. Golubitsky, W. Hao, K.-Y. Lam, Y. Lou, Dimorphism by singularity theory in a model for river ecology, Bull. Math. Biol. 79 (2017), 1051-1069.

[53] R. Gejji, Y. Lou, D. Munther, J. Peyton, Evolutionary convergence to ideal free dispersal strategies and coexistence, Bull. Math Biol. 74 (2012), 257-299.

[54] S. A. H. Geritz, E. Kisdi, G. Meszéna and J. A. J. Metz, Evolutionarily singular strategies and the adaptive growth and branching of the evolutionary tree, Evol. Ecol. 12 (1998), 35-57.

[55] R. Hambrock, Y. Lou, The evolution of conditional dispersal strategy in spatially heterogeneous habitats, Bull. Math. Biol. 71 (2009), 1793-1817.

[56] W. Hao, K.-Y. Lam, Y. Lou, Concentration phenomena in an integro-PDE model for evolution of conditional dispersal, Indiana Univ. Math. J. 68 (2019), 881-923.

[57] W. Hao, K.-Y. Lam, Y. Lou, Ecological and evolutionary dynamics in advective environments: Critical domain size and boundary conditions, submitted, 2020.

[58] A. Hastings, Can spatial variation alone lead to selection for dispersal?, Theor. Pop. Biol. 24 (1983), 244-251.

[59] X. He, K.-Y. Lam, Y. Lou, W.-M. Ni, Dynamics of a consumer-resource reaction-diffusion model: Homogeneous versus heterogeneous environments, J. Math Biol. 78 (2019), 1605-1636.

[60] X. He, W.-M. Ni, The effects of diffusion and spatial variation in Lotka-Volterra competition-diffusion system I: Heterogeneity vs. homogeneity, J. Differential Equations 254 (2013), 528-546.

[61] X. He, W.-M. Ni, The effects of diffusion and spatial variation in Lotka-Volterra competition-diffusion system II: The general case, J. Differential Equations 254 (2013), 4088-4108.

[62] X. He, W.-M. Ni, Global dynamics of the Lotka-Volterra competition-diffusion system: Diffusion and spatial heterogeneity I, Comm. Pure. Appl. Math. 69 (2016), 981-1014.

[63] X. He, W.-M. Ni, Global dynamics of the Lotka-Volterra competition-diffusion system with equal amount of total resources II, Calc. Var. Partial Differential Equations 55 (2016): 25.

[64] P. Hess, Periodic-Parabolic Boundary Value Problems and Positivity, Pitman Research Notes in Mathematics Series 247, Longman Scientific \& Technical, Harlow, 1991.

[65] A.E. Hershey, J. Pastor, B.J. Peterson, G.W. Kling, Stable isotopes resolve the drift paradox for Baetis mayflies in an arctic river, Ecology 74 (1993), 2315-2325.

[66] J. Huisman, M. Arrayas, U. Ebert, B. Sommeijer, How do sinking phytoplankton species manage to persist?, Amer. Naturalist 159 (2002), 245-254.

[67] J. Huisman, P. van Oostveen, F.J. Weissing, Species dynamics in phytoplankton blooms: incomplete mixing and competition for light, Amer. Naturalist 154 (1999), 46-67.

[68] J. Huisman, N.N. Pham Thi, D.K. Karl, B. Sommeijer, Reduced mixing generates oscillations and chaos in oceanic deep chlorophyll, Nature 439 (2006), 322-325.

[69] V. Hutson, W. Shen, G.T. Vickers, Estimates for the principal spectrum point for certain time-dependent parabolic operators, Proc. Amer. Math. Soc. 129 (2000), 1669-1679.

[70] V. Hutson, Y. Lou, K. Mischaikow, P. Poláčik, Competing species near the degenerate limit, SIAM J. Math. Anal. 35 (2003), 453-491.

[71] V. Hutson, K. Mischaikow, P. Poláčik, The evolution of dispersal rates in a heterogeneous time-periodic environment, J. Math. Biol. 43 (2001), 501-533.

[72] S.-B. Hsu, Y. Lou, Single phytoplankton species growth with light and advection in a water column, SIAM. J. Appl. Math. 70 (2010), 2942-2974. 
[73] S.-B. Hsu, K.-Y. Lam, F.-B. Wang, Single species growth consuming inorganic carbon with internal storage in a poorly mixed habitat, J. Math. Biol. 75 (2017), 1775-1825.

[74] J. Inoue, K. Kuto, On the unboundedness of the ratio of species and resources for the diffusive logistic equation, ArXiv preprint, 2020. https://arxiv.org/abs/2001.06308

[75] H. Ishii, I. Takagi, Global stability of stationary solutions to a nonlinear diffusion equation in phytoplankton dynamics, J. Math. Biol. 16 (1982), 1-24.

[76] P.E. Jabin, R. Schram, Selection-Mutation dynamics with spatial dependence, ArXiv preprint, 2020. http://arxiv.org/abs/1601.04553

[77] D. Jiang, K.-Y. Lam, Y. Lou, Z.-C. Wang, Monotonicity and global dynamics of a nonlocal two-species phytoplankton model, SIAM J. Appl. Math. 79 (2019), 716-742.

[78] Y. Jin, R. Peng, J.P. Shi, Population dynamics in river networks, J. Nonlinear Sci. 29 (2019), 2501-2545.

[79] C.Y Kao, Y. Lou, E. Yanagida, Principal eigenvalue for an elliptic problem with indefinite weight on cylindrical domains, Math. Biosci. Eng. 5 (2008), 315-335.

[80] J.T. Kirk, Light and Photosynthesis in Aquatic Ecosystems, Cambridge University Press, Cambridge, UK, 1994.

[81] T. Kolokolnikov, C.H. Ou, Y. Yuan, Phytoplankton depth profiles and their transitions near the critical sinking velocity, J. Math. Biol. 59 (2009), 105-122.

[82] K.-Y. Lam, A semilinear equation with large advection in population dynamics, Thesis (Ph.D.)-University of Minnesota. 2011. 91 pp. ISBN: 978-1124-67112-3, ProQuest LLC

[83] K.-Y. Lam, Concentration phenomena of a semilinear elliptic equation with large advection in an ecological model, J. Differential Equations 250 (2011), 161-181.

[84] K.-Y. Lam, Limiting profiles of semilinear elliptic equations with large advection in population dynamics II, SIAM J. Math. Anal. 44 (2012), 1808-1830.

[85] K.-Y. Lam, Stability of Dirac concentrations in an integro-PDE model for evolution of dispersal, Calc. Var. Partial Differential Equations 56 (2017), no. 3, Paper No. 79, 32 pp.

[86] K.-Y. Lam, Y. Lou, An integro-PDE from evolution of random dispersal, J. Func. Anal. 272 (2017), $1755-1790$.

[87] K.-Y. Lam, Y. Lou, Evolution of dispersal: ESS in spatial models, J. Math. Biol. 68 (2014), 851-877.

[88] K.-Y. Lam, Y. Lou, Evolutionarily stable and convergent stable strategies in reaction-diffusion models for conditional dispersal, Bull. Math. Biol. 76 (2014), 261-291.

[89] K.-Y. Lam, Y. Lou, Persistence, Competition, and Evolution. In: Bianchi A., Hillen T., Lewis M., Yi Y. (eds) The Dynamics of Biological Systems. Mathematics of Planet Earth, vol 4. Springer, Cham, 2019.

[90] K.-Y. Lam, Y. Lou, F. Lutscher, Evolution of dispersal in closed advective environments, J. Biol. Dyn. 9 (2015), 188-212.

[91] K.-Y. Lam, W.-M. Ni, Limiting profiles of semilinear elliptic equations with large advection in population dynamics, Discrete Contin. Dyn. Syst. 28 (2010), 1051-1067.

[92] K.-Y. Lam, W.-M. Ni, Uniqueness and complete dynamics of the Lotka-Volterra competition diffusion system, SIAM J. Appl. Math. 72 (2012), 1695-1712.

[93] K.-Y. Lam, W.-M. Ni, Advection-mediated competition in general environments, J. Differential Equations 257 (2014), 3466-3500.

[94] K.-Y. Lam, Y. Lou, B. Perthame, A Hamilton-Jacobi approach to evolution of dispersal, in preparation.

[95] H. Li, R. Peng, F.-B. Wang, Varying total population enhances disease persistence: qualitative analysis on a diffusive SIS epidemic model, J. Differential Equations 262 (2017), 885-913.

[96] H. Li, R. Peng, T. Xiang, Dynamics and asymptotic profiles of endemic equilibrium for two frequencydependent SIS epidemic models with cross-diffusion, Eur. J. Appl. Math. 31 (2020), 26-56.

[97] R. Li, Y. Lou, Some monotone properties for solutions to a reaction-diffusion model, Discrete Contin. Dyn. Syst. Ser. B 24 (2019), 4445-4455. 
[98] S. Liang, Y. Lou, On the dependence of the population size on the dispersal rate, Discrete Contin. Dyn. Syst. Ser. B 17 (2012), 2771-2788.

[99] S. Liu, Y. Lou, A functional approach towards eigenvalue problems associated with incompressible flow, Discrete Contin. Dyn. Syst. 40 (2020), 3715-3736.

[100] S. Liu, Y. Lou, The roles of frequency and movement in a spatial SIS model with time-periodic environment, submitted, 2020.

[101] S. Liu, Y. Lou, R. Peng, M. Zhou, Monotonicity of the principal eigenvalue for a linear time-periodic parabolic operator, Proc. Amer. Math. Soc. 47 (2019), 5291-5302.

[102] S. Liu, Y. Lou, R. Peng, M. Zhou, Asymptotics of the principal eigenvalue for a linear time-periodic parabolic operator I: Large advection, ArXiv preprint, 2020. https://arxiv.org/abs/2002.01330.

[103] S. Liu, Y. Lou, R. Peng, M. Zhou, Asymptotics of the principal eigenvalue for a linear time-periodic parabolic operator II: Small diffusion, ArXiv preprint, 2020. https://arxiv.org/abs/2002.01357.

[104] S. Liu, Y. Lou, P. Song, A new monotonicity for principal eigenvalues with applications to time-periodic patch models, submitted, 2020.

[105] Y. Lou, On the effects of migration and spatial heterogeneity on single and multiple species, J. Differential Equations 223 (2006), 400-426.

[106] Y. Lou, S. Martinez, P. Poláčik, Loops and branches of coexistence states in a Lotka-Volterra competition model, J. Differential Equations 230 (2006), 720-742.

[107] Y. Lou, Some challenging mathematical problems in evolution of dispersal and population dynamics, Tutorials in mathematical biosciences. IV, 171-205, Lecture Notes in Math., 1922, Math. Biosci. Subser., Springer, Berlin, 2008.

[108] Y. Lou, Some reaction-diffusion models in spatial ecology (in Chinese), Sci. Sin. Math 45 (2015), 16191634.

[109] Y. Lou, On several partial differential equation models in population dynamics (in Chinese), to appears in the Lecture Series of Institute for Mathematics, Chinese Academy of Sciences.

[110] Y. Lou, F. Lutscher, Evolution of dispersal in advective environments, J. Math Biol. 69 (2014), 1319-1342.

[111] Y. Lou, D. Munther, Dynamics of a three species competition model, Discrete Contin. Dyn. Syst. 32 (2012), 3099-3131.

[112] Y. Lou, D. Xiao, P. Zhou, Qualitative analysis for a Lotka-Volterra competition system in advective homogeneous environment, Discrete Contin. Dyn. Syst. 36 (2016), 953-969.

[113] Y. Lou, B. Wang, Local dynamics of a diffusive predator-prey model in spatially heterogeneous environment, J. Fixed Point Theory Appl. 19 (2017), 755-772.

[114] Y. Lou, X.-Q. Zhao, P. Zhou, Global dynamics of a Lotka-Volterre competition-diffusion-advection system in heterogeneous environments, J. Math. Pure. Appl. 121 (2019), 47-82.

[115] Y. Lou, P. Zhou, Evolution of dispersal in advective homogeneous environments: The effect of boundary conditions, J. Differential Equations 259 (2015), 141-171.

[116] F. Lutscher, E. Pachepsky, M.A. Lewis, The effect of dispersal patterns on stream populations, SIAM Rev. 47 (2005), 749-772.

[117] F. Lutscher, E. McCauley, M.A. Lewis, Effects of heterogeneity on spread and persistence in rivers, Bull. Math. Biol. 68 (2006), 2129-2160.

[118] M.J. Ma, C.H. Ou, Existence, uniqueness, stability and bifurcation of periodic patterns for a seasonal single phytoplankton model with self-shading effect, J. Differential Equations 263 (2017), 5630-5655.

[119] J. Maynard Smith, G. Price, The logic of animal conflict, Nature 246 (1973), 15-18.

[120] P. Magal, G. F. Webb, Y. Wu, On the basic reproduction number of reaction-diffusion epidemic models, SIAM J. Appl. Math. 79 (2019), 284-304.

[121] I. Mazari, G. Nadin, Y. Privat, Optimal location of resources maximizing the total population size in logistic models, J. Math. Pure. Appl. 134 (2020), 1-35. 
[122] I. Mazari, G. Nadin, Y. Privat, Shape optimization of a weighted two-phase Dirichlet eigenvalue, ArXiv preprint, 2020. https://arxiv.org/abs/2001.02958.

[123] I. Mazari, D. Ruiz-Balet, A fragmentation phenomenon for a non-energetic optimal control problem: optimisation of the total population size in logistic diffusive models, preprint, 2020. https://hal.archivesouvertes.fr/hal-02523753

[124] L. Mei, X.Y. Zhang, Existence and nonexistence of positive steady states in multi-species phytoplankton dynamics, J. Differential Equations 253 (2012), 2025-2063.

[125] S. Mirrahimi, B. Perthame, Asymptotic analysis of a selection model with space, J. Math. Pures Appl. 104 (2015), 1108-1118.

[126] K. Müller, Investigations on the organic drift in North Swedish streams, Rep. Inst. Freshw. Res. Drottningholm 35 (1954), 133-148.

[127] K. Nagahara, Y. Lou, E. Yanagida, Maximizing the total population in a patchy environment, submitted, 2020.

[128] K. Nagahara, E. Yanagida, Maximization of the total population in a reaction-diffusion model with logistic growth, Calc. Var. Partial Differential Equations 57 (2018): 80.

[129] W.-M. Ni, The Mathematics of Diffusion, CBMS Reg. Conf. Ser. Appl. Math. 82, SIAM, Philadelphia, 2011.

[130] W.-M. Ni, private communication.

[131] W. Ni, J. Shi, M. Wang, Global stability of nonhomogeneous equilibrium solution for the diffusive LotkaVolterra competition model, ArXiv preprint, 2020. http://arxiv.org/abs/1909.03376

[132] S. Nordmann, B. Perthame, Dynamics of concentration in a population structured by age and a phenotypic trait with mutations. Convergence of the corrector, ArXiv preprint, 2020. http://arxiv.org/abs/2001.04323v1.

[133] R. Peng, Asymptotic profiles of the positive steady state for an SIS epidemic reaction-diffusion model I, J. Differential Equations 247 (2009), 1096-1119.

[134] R. Peng S. Liu, Global stability of the steady states of an SIS epidemic reaction-diffusion model, Nonlinear Anal. 71 (2009), 239-247.

[135] R. Peng, F. Yi, Asymptotic profile of the positive steady state for an SIS epidemic reaction-diffusion model: effects of epidemic risk and population movement, Phys. D 259 (2013), 8-25.

[136] R. Peng, X.Q. Zhao, A reaction-diffusion SIS epidemic model in a time-periodic environment, Nonlinearity 25 (2012), 1451-1471.

[137] R. Peng, X.-Q. Zhao, Effects of diffusion and advection on the principal eigenvalue of a periodic parabolic problem with applications, Calc. Var. Partial Differential Equations 54 (2015), 1611-1642.

[138] R. Peng, X.-Q. Zhao, A nonlocal and periodic reaction-diffusion-advection model of a single phytoplankton species, J. Math. Biol. 72 (2016), 755-791.

[139] R. Peng, M. Zhou, Effects of large degenerate advection and boundary conditions on the principal eigenvalue and its eigenfunction of a linear second order elliptic operator, Indiana Univ. Math J. 67 (2018), 2523-2568.

[140] B. Perthame, P.E. Souganidis, Rare mutations limit of a steady state dispersion trait model, Math. Model. Nat. Phenom. 11 (2016), 154-166.

[141] N. Shigesada, A. Okubo, Analysis of the self-shading effect on algal vertical distribution in natural waters, J. Math. Biol. 12 (1981), 311-326.

[142] H. Smith, Monotone Dynamical Systems, Mathematical Surveys and Monographs 41, American Mathematical Society, Providence, Rhode Island, U.S.A. 1995

[143] P. Song, Y. Lou, Y. Xiao, A spatial SEIRS reaction-diffusion model in heterogeneous environment, J. Differential Equations 267 (2018), 5084-5114. 
[144] D.C. Speirs, W.S.C. Gurney, Population persistence in rivers and estuaries, Ecology 82 (2001), 12191237.

[145] N. Tuncer, M. Martcheva, Analytical and numerical approaches coexistence of strains in a two-strain SIS model with diffusion, J Biol. Dyn. 6 (2012), 406-439.

[146] P. van den Driessche, J. Watmough, Reproduction numbers and sub-threshold endemic equilibria for compartmental models of disease transmission, John A. Jacquez memorial volume, Math. Biosci. 180 (2002), 29-48.

[147] O. Vasilyeva, Population dynamics in river networks: analysis of steady states, J. Math. Biol. 79 (2019), 63-100.

[148] O. Vasilyeva, F. Lutscher, Population dynamics in rivers: analysis of steady states, Can. Appl. Math. Quart. 18 (2011), 439-469.

[149] W. Wang, X.-Q. Zhao, A nonlocal and time-delayed reaction-diffusion model of dengue transmission, SIAM J. Appl. Math. 71 (2011), 147-168.

[150] W.-D. Wang, X.-Q. Zhao, Basic reproduction numbers for reaction-diffusion epidemic models, SIAM J. Appl. Dyn. Syst. 11 (2012), 1652-1673.

[151] Y. Wang, J.P. Shi, Persistence and extinction of population in reaction-diffusion-advection model with weak Allee effect growth, SIAM J. Appl. Math. 79 (2019), 1293-1313.

[152] Y. Wang, J.P. Shi, J.F. Wang, Persistence and extinction of population in reaction-diffusion-advection model with strong Allee effect growth, J. Math. Biol. 78 (2019), 2093-2140.

[153] Y. Wu, N. Tuncer, M. Martcheva, Coexistence and competitive exclusion in an SIS model with standard incidence and diffusion, Discrete Contin. Dyn. Syst. Ser. B 22 (2017), 1167-1187.

[154] Y. Wu, X. Zou, Asymptotic profiles of steady states for a diffusive SIS epidemic model with mass action infection mechanism, J. Differential Equations 261 (2016), 4424-4447.

[155] J.-J. Xiang, Y. Fang, Evolutionarily stable dispersal strategies in a two-patch advective environment, Discrete Contin. Dyn. Syst. Ser. B 24 (2019), 1875-1887.

[156] F. Xu, W. Gan, D. Tang, Population dynamics and evolution in river ecosystems, Nonlinear Anal. Real World Appl. 51 (2020): 102983.

[157] X.-Q. Zhao, P. Zhou, On a Lotka-Volterra competition model: the effects of advection and spatial variation, Calc. Var. Partial Differential Equations 55 (2016): 73.

[158] P. Zhou, On a Lotka-Volterra competition system: diffusion vs advection, Calc. Var. Partial Differential Equations 55 (2016): 137. 